New Developments in the Theory of HTSC

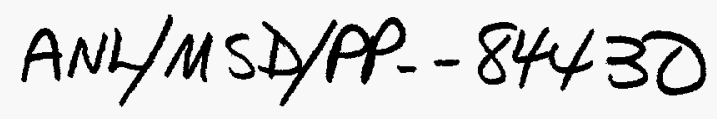

\author{
A. A. Abrikosov \\ Materials Science Division \\ Argonne National Laboratory \\ Argonne, IL 60439
}

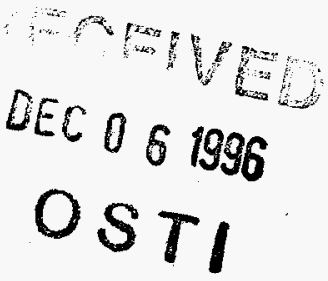
$\therefore$ The submitted manuscript has been authored by a
contractor of the U.S. Government Under contract No. W-
31-109-ENG-38. Accordingly, the U.S. Government
retains a nonexclusive, royalty-free license to publish or
reproduce the publishod form of this contribution, or allow
ofhers to do so for U.S Government pumposes

September, 1994

$/ \mathrm{sm}$

Distribution:

1-2. M. J. Masek

3. B. D. Dunlap

4. G. W. Crabtree

5. A. A. Abrikosov

6. Editorial Office

7. Authors

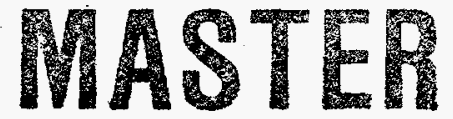

This work is supported by the Division of Materials Sciences, Office of Basic Energy Sciences of DOE, under contract No. W-31-109-ENG-38. 


\section{DISCLAIMER}

This report was prepared as an account of work sponsored by an agency of the United States Government. Neither the United States Government nor any agency thereof, nor any of their employees, make any warranty, express or implied, or assumes any legal liability or responsibility for the accuracy, completeness, or usefulness of any information, apparatus, product, or process disclosed, or represents that its use would not infringe privately owned rights. Reference herein to any specific commercial product, process, or service by trade name, trademark, manufacturer, or otherwise does not necessarily constitute or imply its endorsement, recommendation, or favoring by the United States Government or any agency thereof. The views and opinions of authors expressed herein do not necessarily state or reflect those of the United States Government or any agency thereof. 


\section{DISCLAIMER}

Portions of this document may be illegible in electronic image products. Images are produced from the best available original document. 


\title{
NEW DEVELOPMENTS IN THE THEORY OF HTSC
}

\author{
A. The Layered S-N Model, B. Extended Saddle Point Singularities and \\ Long Range Interaction (E-L Model) , C. Reconciliation on the Nature of \\ the Order Parameter
}

\author{
A.A.ABRIKOSOV, \\ Materials Science Division, Argonne National Laboratory, bldg.223, \\ 9700 South Cass Avenue, Argonne, IL 60439
}

\begin{abstract}
A.The superconductor is supposed to consist of alternating layers of two kinds: (1) layers with an attractive electron interaction and an effective mass of usual magnitude, (2) layers without interaction and with a large effective mass. The overlap between the layers is assumed to be small, its energy, $t$, being much less than $\Delta$. It is shown, that such a model explains the most peculiar property found in experiments on electronic Raman light scattering in BSCCO 2212: different threshold values for the Raman satellite measured at two different polarizations of the incident and scattered light.

The tunneling conductance $G(V)=d J / d V$ is analyzed for the same model. In order to fit the qualitative features of experimental data, it is assumed that the tunneling probability to the normal layers is much less, than to the superconducting layers. The conductance is calculated for the case $t \ll \Delta$. A brief analysis is given for the case $t \sim \Delta$, which proves that such an assumption definitely contradicts the experimental data for BSCCO. The possible nature of the electronic states in the normal layers is discussed.

B. In connection with the experimental discovery (angle resolved photoemission spectroscopy, ARPES) of the extended saddle point singularities in the electron spectrum of a variety of HTSC consequences are derived for $T_{c}$ and $\Delta$ in a simple model. A large enhancement of superconductivity is possible if the singularity has a sufficient extension and is located close to the Fermi energy.

In order to explain the anisotropy of the energy gap, observed in ARPES experiments, on the basis of the "extended saddle point singularities" an assumption is done that the Coulomb interactions are weakly screened, i.e. the Debye screening radius is much larger than the lattice period; this makes the electron interaction long ranged ( $\mathrm{E}-\mathrm{L}$ model). The consequence of this model is the change of the isotope effect with composition and also the change of $T_{C}$. The idea is that if the energy difference between the Fermi level and the saddle point is less than the Debye energy, this distance defines the effective energy scale, and hence there is a small isotope effect, whereas in the opposite case the Debye energy defines the cut-off, and the usual isotope effect is restored. Simultaneously $\mathrm{T}_{\mathrm{C}}$ decreases.
\end{abstract}


It also follows that at low temperatures the nomal state resistivity is mostly defined by electron-electron scattering, and its temperature dependence is $\rho \propto T$.

C. Several data on the phase determination by single and multiple Josephson junctions, and on the temperature dependence of the penetration depth are in favor of an order parameter, changing its sign as function of momentum, and, consequently, the energy gap having nodes. These data can be incorporated in the model described above if, apart from long-ranged phonon mediated attraction, a short range repulsion of another origin is assumed (E-L-U model).

\section{ELECTRONIC RAMAN SCATTERING [1]}

The problem of Raman light scattering from electrons in HTSC became very important, since these results are critical in defining what kind of pairing takes place: ordinary BCS-type pairing, or something more complicated. One of the most important points is whether or not the energy gap vanishes at certain points or contours along the Fermi surface. In the latter case BCS-type pairing is possible (although not necessarily achieved by phonons). Vanishing of the gap somewhere along the Fermi surface would require non-BCS-type pairing.

Raman scattering is a contactless experiment. The laser spot may be very small so that a good single-domain piece of the surface is selected. It is possible, that the properties of the surface are different from those of the bulk, but the penetration depth of light is of the order of $10^{3} \mathrm{~A}$, so is one expects that bulk properties are being measured

According to the theory for an anisotropic metal $[2,3]$ the Raman satellite forms a wide band starting from $\omega-\omega^{\prime}=2 \Delta_{\min }$ with a sharp increase from zero to some maximal value in the region $\omega-\omega^{\prime}-24 \min ^{-\bar{\Delta}}$ and then decreases with $\omega$ (as $\left(\omega-\omega^{\prime}\right)^{-2}$ for a short coherence length . In various experiments (see references in [3]) with different substances the sattelite starts at $\omega-\omega^{\circ}=0$ with a linear dependence $d \sigma \sim \omega-\omega^{\prime}$ and then follows essentially the theoretical predictions. This could mean that the energy gap turned to zero along lines, which could be possible due to the cylindrical shape of the Fermi surface in a quasi-2-d metal. However in the experiments by Boekholt, Hoffmann and Guentherodt [4], a definite energy

gap was observed. They studied very perfect single crystals of

$\mathrm{Bi}_{2} \mathrm{Sr}_{2} \mathrm{CaCl}_{2} \mathrm{O}_{8+\delta}$ with a surface thoroughly characterized by a highresolution electron microscope, and the temperature of the laser spot was well controlled by comparison of intensities of the Stokes and anti-Stokes satellites. An anisotropic gap was observed with $2 \Delta_{/ /} / T_{c}=5.7,2 \Delta_{\perp}$ $r_{C}=3.4$, where $\Delta /$ means an experiment in the $Z(X X) \bar{Z}$ geometry and 
$\Delta$

a $\mathrm{Y}(\mathrm{ZZ}) \overline{\mathrm{Y}}$ geometry (here $\mathrm{Z}(\mathrm{XX}) \overline{\mathrm{Z}}$ means that the direction of

incidence is along $Z$ - the main axis, the scattered beam is observed in the backward direction and both polarizations are along $X$ in the plane). Since this definitely contradicted our previous theoretical predictions $[2,3]$ it created doubts in the applicability of the model of an anisotropic metal which we have used.

Recently, models taking directly into account the layered structure of the HTSC have become popular. These models can explain some properties of the vortex structure in the mixed state, pinning and the current-voltage characteristic. Tachiki et al.(TTSA) [5] have shown, that such a model explains qualitative features of the tunneling conductance; and the present study was carried out to learn whether a model of this type could explain, at least qualitatively, the the Raman scattering results. It was shown, that this

is really the case. Even the fact that $\Delta_{j}$ is almost twice the observed $\Delta_{\perp}$ becomes understandable. In the calculations we supposed $\mathrm{T}=0$.

In the TTSA model it was assumed, that the superconductor consists of 5 periodically repeated layers of which layers 1 and 5 have an electron attraction leading to Cooper pairing, whereas layers 2,3 and 4 have no such attraction and are normal. There is hopping between adjacent layers. Here our task is to study qualitative features of the electronic Raman scattering and to find an explanation of the main observed properties; vizi dependence on polarization and appearance of two different gaps. We did not find it very useful to seek exact numerical agreement with experiment taking a many-layered Hamiltonian with many adjustable parameters, and considered a model with only two alternating kinds of layers: (1) superconducting with an attractive electron interaction and (2) normal with no interaction (Fig.1).

Fig. 1. Layered S-N models of a High- $\mathrm{T}_{\mathrm{C}}$ Superconductor

Our Hamiltonian has the form

$$
H=H_{1}+H_{2}+T+I \text {. }
$$

Here $H_{1,2}$ are the bare Hamiltonians of the $S$ - and $\mathrm{N}$-planes, $T$ is the hopping part, and I - the BCS interaction in the S-planes. The $\psi$-operators entering $\mathrm{H}$ are of the tight binding type

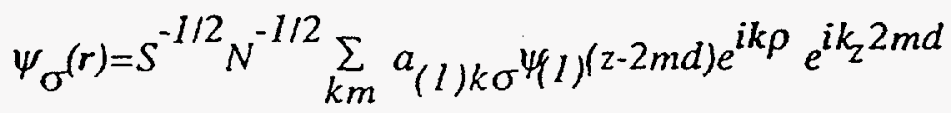




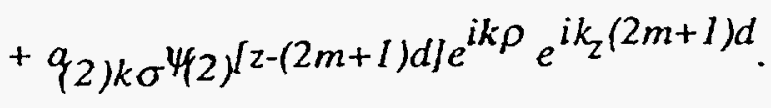

Here $k=\left(k, k_{z}\right), S$ is the normalization area in the plane, $N$ - the number of periods along $z, \psi_{(1)}$ and $\psi_{(2)}$ are the localized wave functions of the superconducting and normal layers, and the $a_{(i) k \sigma}$ - the corresponding annihilation operators. We assume that $H_{I}$ has only diagonal matrix elements between $\psi_{(1)} e^{i k \rho}$ with the same $m$, and they correspond to the kinetic energy in the $S$-planes, and similarly for $\mathrm{H}_{2}$ for the $\mathrm{N}$-planes. The hopping part, $T$, has off-diagonal matrix elements between $\psi_{(1)}$ and $\Psi_{(2)}$, with adjacent m's and the same $\sigma$ 's which are equal to $t / 2$ (it can be said, that the operator $T$ transforms $\psi_{(1)}(z-2 m d)$ into $\left.\psi_{(2)}\right)^{(z-(2 m+1) d]}$ and vice versa). The interaction part I has matrix elements equal to $-\mathrm{g}$ only in the same superconducting layer.

Substituting (2) into $H-N \mu$ we get

$$
\begin{aligned}
& H-N \mu=\sum_{k \sigma}\left[a_{(1) k \sigma^{+}}^{+}(k) a_{(1) k \sigma^{2}}+a_{(2) k \sigma^{+}} \xi_{(k) a_{(2) k \sigma}}\right. \\
& +t \cos k d\left(a_{(1) k \sigma^{+}}^{a}(2) k \sigma^{+a^{+}}(2) k \sigma^{a}(1) k \sigma^{)]}\right. \\
& -\frac{g}{S N} \sum_{k_{1}+k_{2}-k_{3}-k_{4}=0} a_{(1) k_{1}-}^{+} a_{(1) k_{2}+q_{(1) k_{3}+}^{+}}^{+}(1) k_{4-} \text {; }
\end{aligned}
$$

indices $\sigma=t,-$ refer to the spin projections, $\xi_{I 2}=v_{12}\left(k-k_{o}\right)$. In the self-consistent field approximation.we substitute the interaction term by

$$
-\Delta^{*} \sum_{k} q_{(1) k+q(1)-k-}-\Delta \sum_{k} a_{(1)-k-}^{+} a_{(1) k+}^{+}
$$

where

$$
\Delta=\frac{g}{S N} \sum_{k}<q(1) k+q(1)-k->
$$

We shall make the following assumptions: $t \propto \Delta \sqrt{\beta}, \beta=m_{1} / m_{2} \approx I$. Then the eigenvalues are

$$
\begin{aligned}
& q \equiv\left[\xi_{1}^{2}+\Delta^{2}+2 t^{2}\left(k_{z}\right)\right]^{I / 2}, \varepsilon_{2} \cong\left[\xi_{2}^{2}+t^{4}\left(k_{z}\right) / \Delta^{2}\right]^{I / 2} \\
& \xi=-\varepsilon_{2}, \xi_{4}=-\xi_{1} ; t\left(k_{2}\right)=t \cos k_{2} d
\end{aligned}
$$


(the reason for leaving the term $2 t^{2}$ in $\varepsilon_{2}$ and $\varepsilon_{4}$ is its dependance on $k_{\mathrm{Z}}$ ). At the first glance there are two finite gaps. However since $t\left(k_{z}\right)$ $=t \cos \left(k_{Z} d\right)$ the second gap vanishes at the boundaries of the Brillouin zone: $k_{z} \rightarrow \pi / 2 d$ or $k_{z} \rightarrow-\pi / 2 d$.

In the (ZZ) geometry the scattering is defined by the $A_{2}$ component of the vector potential. In the tight-binding model it enters the wave functions through the factors

$$
\exp \left(-i \frac{e}{c} \int^{z} A_{z} d z\right)
$$

therefore if $\psi$ and $\psi^{+}$appear with the same argument, the factors are cancèled out, and the only terms in the Hamiltonian containing $A_{z}$ are the hopping terms. They contain factors of the type

$$
\exp \left( \pm \frac{i e}{c} \int_{2 m d}^{(2 m+1) d} A_{z} d z\right)
$$

and assuming that $A_{\mathrm{z}}$ doesn't vary noticeably at atomic distances these. factors can be replaced by

$$
\exp \left[ \pm \frac{i e d}{c} A_{2}(2 m d)\right]
$$

The result is that in the hopping terms $k_{z}$ are replaced by $k_{z}-(e / c) A_{z}$.

Omitting the detailes of calculations and formulas, the final answer can be illustrated by Fig. 2 .

Fig. 2. Electronic Raman scattering cross-section for the geometry $\mathrm{X}(\mathrm{ZZ}) \overline{\mathrm{X}}$ 
The scattering is accompanied by hopping of the electron between $N$ and $S$ planes. The threshold is therefore $\Delta$, as in

tunneling between a normal and superconducting metal. After a logarithmic singularity at $\omega-\omega^{\prime}=\Delta+2 t^{2} / \Delta$, which is the maximum of the sum of the gaps in both bands (in the sence of the dependence on $k_{\mathrm{Z}}$; the bands are assumed to be isotropic in the plain), the cross section decreases as $[\Delta(\omega-$ $\left.\left.\omega^{\prime}\right)\right]^{2}$. The average scale for reasonable values of parameters is $d \sigma \sim 10^{-12} \frac{d \omega}{\Delta} d \Omega$.

In the case of parallel polarisation $\mathrm{Z}(\mathrm{XX}) \overline{\mathrm{Z}}$ the interaction of electrons with light appears in the kinetic energy terms of the Hamiltonian and is given by

$$
\left.\int \stackrel{+}{+} \psi_{\sigma}^{(}-\frac{e}{c} v_{1 x} A_{x}+\frac{e^{2}}{2 m_{1} c^{2}} A_{x}^{2}-\frac{e}{c} v_{2 x} A_{x}+\frac{e^{2}}{2 m_{2} c^{2}} A_{x}^{2}\right) \psi_{\sigma} d V
$$

As was shown in [2], the terms linear in $A_{x}$ contribute only minor corrections to $d \sigma$, and so we consider only the quadratic terms. They contain the band mass in the denominator. In order to obtain a finite threshold value of $\omega-\omega^{\prime}$. we have to assume that $m \geqslant m$. Then the main contribution is due to the superconducting layers. The result can be illustrated by Fig. 3 .

fig. 3. Electronic Raman scattering cross-section for the geometry $Z(X X) \bar{Z}$

The threshold is at $2 \Delta$. A discontinuous jump (it can be smoothed out, if the anisotropy in the plane is taken into account) is followed by a logarithmic singularity at the maximum of the double gap $2\left(\Delta+t^{2} / \Delta\right)$ and then falls off as $\left[\Delta /\left(\omega-\omega^{\prime}\right)\right]^{2}$. The scale is again of the order $d \sigma \sim 10^{-12} \frac{d \omega^{\prime}}{\Delta} d \Omega$. At smaller $\omega-\omega^{\prime}<2 \Delta$ there can be a small contribution from the normal band but other sources of additional scattering cannot be excluded. 
According to this theory the threshold value for $d \sigma /$ should be exactly twice the one for $d \sigma_{\perp}$. The experimental result is not exactly the same : $2 \Delta_{/ /} / T_{c}=5.7,2 \Delta_{\perp} / T_{c}=3.4$. There is, however, a simple way to correct this difference. We have assumed that there is no interaction of electrons in the "normal" planes. But this is quite impossible. So let us assume that there is a very small interaction, $g_{2}$. Then it is natural to beleive that a condensate is formed, and hence a nonvanishing $<a_{(2) k+q(2)-k_{-}}>$exists. If we assume $\Delta_{2}>0$ the threshold will become $\Delta_{\perp}=\Delta+\Delta_{2}$, and this could explain why $\Delta_{\perp}$ is not equal to $\Delta_{/ /} / 2$.

From the experimental values quoted above we obtain $\Delta T_{C}=2.85$,

$\Delta_{2} i T_{\mathrm{C}}=0.55$, i.e. $\Delta$ is more than five times larger than $\Delta$.

\section{TUNNELING CONDUCTANCE [6]}

Another method of direct measurement of the energy spectrum and particularly of the energy gap is the tunneling conductance $G=d J / d V$.

as a function of the voltage $V$. It is well known that $G$ is proportional to the electronic density of states. Here we meet a paradox. According to the last measurements of Hasegawa [7] the main contribution comes from the superconducting layers, and a clear gap of the order of the one obtained

from Raman experiments on $\mathrm{Bi}_{2} \mathrm{Sr}_{2} \mathrm{CaCl}_{2} \mathrm{O}_{8+\delta}$ is seen. On the other hand the tunneling conductance reflects the electronic density of states which is proportional to the effective mass in any dimensionality. Since we had to assume for interpretation of the Raman experiments that the effective mass of the normal layers is much larger than the mass of the superconducting layers, the contribution of the normal layers to the density of states would dominate in contradiction with the experiment. There is a way to resolve this paradox assuming the tunneling probabilities to the $S$ - and $N$-layers to be very different. We leave the detailed discussion of the possible origin to the end of this part.

We consider a contact between some normal metal and the S-N superconductor. According e.g. to ref.[8], § 22.3, we have

$$
\begin{aligned}
& \mathrm{J}=2 \pi e(2 d)^{-1} \sum_{i=12 ; p q} T_{p q}^{(i)} \sum_{m} \delta\left(E_{o}-E_{m}\right)\left\{\left(\left(A_{p i}^{+} b_{q+}\right)_{m}\left(A_{p l} b_{q+}^{+}\right\}_{m o}\right.\right. \\
& +\left(A_{p i}, b_{q-}\right)_{o m}\left(A_{p i}^{+} b_{q-}^{+}\right)_{m o}^{-(}\left(A_{p i} b_{q+}^{+}\right)_{o m}\left(A_{p i}^{+} b_{q+} b_{m o}\right. \\
& -\left(A_{p i}^{+}, b_{q-}^{+}\right)_{o m}\left(A_{p i}, b_{q-}\right)_{m o}{ }^{3}
\end{aligned}
$$


The factor $(2 d)^{-1}$ was introduced to define the current density. We assume that the phases of tunneling amplitudes of the superconducting ( $i=1)$ and normal $(i=2)$ layers are uncorrelated. The operators $b_{q \sigma}$ correspond to the normal metal. The operators for the superconductor are the same, as used previously, but rewritten in the Nambu representation: namely the $A_{p i}$ mean annihilation of quasiparticles in layers $i=I(\mathrm{~S})$ and $i=2(\mathrm{~N})$ with momentum $p$ and spin 1/2; the $A_{p i}$ - mean creation of quasiparticles with momentum $-p$ and spin $-1 / 2$. The temperature is taken to be zero, the index zero means the ground state, summation is taken over the final states $m$.

After that we pass over to the band representation with the energy levels $E_{1}=\varepsilon_{1}, E_{2}=\varepsilon_{2}, E_{3}=-\varepsilon_{2}, E_{4}=-\varepsilon_{1}$. We will assume the tunneling probability to the $\mathrm{N}$-layers to be much smaller than to the $\mathrm{S}$-layers

$$
\overline{\left|T^{(2)}\right|^{2}} \times \beta \overline{\left|T^{(1)}\right|^{2}}, \beta=m_{1} / m_{2} \ll 1,
$$

and first consider the case of small hopping $t \propto \Delta \sqrt{\beta}$.

The result for this case is

$$
\begin{aligned}
& J=4 \pi e(2 d)^{-1} \frac{\rho_{n}}{2} \frac{m_{I}}{2 \pi} \int_{0}^{\infty} d \xi \frac{2}{\pi} \int_{0}^{1} \frac{d q}{(1-q)} \\
& \times_{\{I} \overline{\left|T^{(1)}\right|^{2}} \cdot\left(\frac{t q}{\Delta}\right)^{2}+\overline{\left|T^{(2)}\right|^{2}} \cdot \mid\left(n_{\varepsilon_{2}-e V}{ }^{-n} \varepsilon_{2}+e V\right)
\end{aligned}
$$

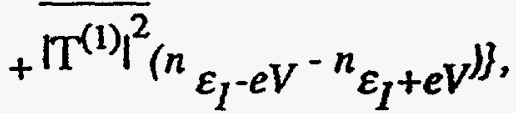

where $n_{\varepsilon}$ are the Fermi functions of corresponding arguments, $\xi=v_{1}(|k|$. $\left.k_{o}\right), q=\cos k_{z} d$. The contribution from the band (2) is small coming either from small tunneling to the $\mathrm{N}$-layers or from the small hybridization with the S-layers. Nevertheless it is of some importance since in the conductance $G=d J / d V$ it is the only one at $e V<\Delta$. The shape of the curve $G(V)$ is presented at Fig.4. We have a jump at the threshold $\mathrm{eV}=\Delta$ followed by a logarithmic singularity at the maximal gap of the $f$ band and a subsequent decrease to the normal state value. There is a small contribution of the $\varepsilon_{2}$ band at $e V<\Delta$ but in experimental conditions it may be obscured by surface defects or other phenomena. 
Fig. 4. Tunneling conductance for small hopping, $t \times \Delta \sqrt{\beta}$

For generality we considered also the case $t \sim \Delta$, since in the S-N model the assumption $t * \Delta$ leads to suppression of superconductivity [9]. The result is plotted at Fig.5.

Fig. 5. Tunneling conductance for large hopping, $t \sim \Delta$

As the voltage is increased from zero, the conductance $G$ starts with the value $I / R$, corresponding to a normal metal, at voltages much less than $\Delta / e$. After a smooth maximum it drops discontinuously at $V_{2}$ to a very small value; then at $V_{0}$ it jumps up again to a finite value on the order of $I / R$. After that, it has a logarithmic singularity at $V_{1}$, and at still larger voltages it tends to $1 / R$. In experiment, a dip is always found around $V=0$, and although a small maximum is sometimes seen at very low voltages, it never reaches values comparable to the main maximum, which occurs at $e V_{\text {max }}=$ $a T_{c}$ with $a \sim 3$. This all makes it very unlikely that the $S-N$ layered model with $t \sim \Delta$ is good for BSCCO. On the other hand, the assumption

$t \ll \Delta \sqrt{\beta}$ together with $\overline{\left|\mathrm{T}^{(2)}\right|^{2}} \ll \beta \overline{\mid \mathrm{T}^{\left.(1)\right|^{2}}}$ leads to results resembling the experimental data (see e.g. ref. [6]).

Now we retrurn to our basic assumption The only way to achieve agreement of the model with the tunneling data is to assume 
$\overline{\left|T^{(2)}\right|^{2}} \ll \beta \mid \overline{\left|T^{(1)}\right|^{2}}$, and this means that the states of electrons in the $N$-layers have to be different from those in the S-layers. If one thinks more thoroughly about the $\mathrm{S}-\mathrm{N}$ model, one encounters another assumption, which looks rather strange. In the original Hamiltonian (3) it is implicitly supposed that the Fermi-circles in the normal and superconducting layers are exactly the same. Otherwise the energy and momentum conservation laws would be violated. But this means equal number of electrons (or holes) in both types of layers, which is unlikely. If there were a pronounced anisotropy in one of the layers (as in YBCO) that would not be necessary, since the Fermi surfaces could just cross at some points. But in BSCCO there is no substantial anisotropy.

There is a possible way to resolve these contradictions, namely to assume that the electrons in the $\mathrm{N}$-layers form a continuous band of localized states (as occurs in Anderson localization). Then there is no momentum conservation. Such an assumption is compatible with small tunneling matrix elements, since for effective tunneling a rapid change of occupancy of the surface states is necessary, and if the states are essentially localized, this happens very slowly.

In this connection it should be mentioned that the situation in the 1-2-3 substances is entirely different. A model of two types of superconducting layers, one of them being isotropic and the other quasi-1-dimensional, looks more appropriate.

\section{EXTENDED SADDLE POINT SINGULARITIES [10].}

Usually the angle resolved photoemission spectroscopy (ARPES) is applied for determination of the Fermi surface in cases where the usual methods, e.g. de Haas - van Alfen, or Gantmakher oscillatory effects in thin single crystalline films, fail for some reason. Such a situation happens in the high $T_{c}$ cuprates, because the normal state corresponds to such high temperatures that all the oscillations vanish, and destruction of superconductivity by magnetic field at lower temperatures requires tremendously large magnetic fields. J.C.Campuzano and K.Gofron from Argonne managed to increase the resolution of their experiments to such an extent that they were able to find not only the Fermi surface but. also the electron energy spectrum in the vicinity of the Fermi energy. Since the cuprates are quasi-2D substances, the energy depends very weakly on one of the components of the quasimomentum, say $k_{z}$, and can be considered mostly as function of $\boldsymbol{k}$ - the quasimomentum in the a-b plane. They discovered that this dependence has not simple saddle points but what they called "extended saddle points" - rather long regions in the $k$ plane, where the energy depends only on one component of the momentum. These regions are confined to the boundaries of the Brillouin zone. Their location and the curves illustrating the function $\varepsilon\left(k_{x}, k_{y}\right)$ are given at Fig.6, 7. 
Fig. 6. Location of the extended saddle point singularities in the $\left(k_{x}, k_{y}\right)$ plane for $\mathrm{YBa}_{2} \mathrm{Cu}_{3} \mathrm{O}_{7-\delta}$

Fig. 7. The function $\varepsilon\left(k_{x}, k_{y}\right)$ near the singularity

Such singularities were found in $\mathrm{YBa}_{2} \mathrm{Cu}_{3} \mathrm{O}_{6.9}, \mathrm{YBa}_{2} \mathrm{Cu}_{4} \mathrm{O}_{8}$ and $\mathrm{Bi}_{2} \mathrm{Sr}_{2} \mathrm{CaCu}_{2} \mathrm{O}_{8}+\delta$ This discovery was confirmed later by the Srtanford group (Shen, Dessau et al.) [11] and by numerical determinations of the band structure in the same substances as well as in $\mathrm{HgBa}_{2} \mathrm{CuO} 4+\delta$ and $\mathrm{HgBa}_{2} \mathrm{CaCu}_{2} \mathrm{O}_{6}+\delta[12]$

Even a simple saddle point leads to the increase of the density of states, and to enhancement of superconductivity [13]. The extended saddle point means actually that the substance is not only quasi-2D, but quasi-1D with the density of states in a rather wide energy range given by the formula

$$
v(\varepsilon)=\frac{P_{y 0}\left(2 m_{x}\right)^{I / 2}}{(2 \pi)^{2} d\left(\varepsilon-\varepsilon_{o}\right)^{I / 2}},
$$

where $P_{y 0}$ is the extension of the singularity (we assume here that it goes along $\left.k_{y}\right), m_{x}$ is the effective mass for $k_{x}, d$ is the period along the caxis, $\varepsilon_{o}$ the energy of the singularity. Substitution into the self-consistency equation of the BCS theory gives in the limiting cases: 


$$
T_{c}=\frac{8 \gamma}{\pi}\left(\mu-\varepsilon_{o}\right) \exp \left[\frac{2^{1 / 2 \pi^{2} d\left(\mu-\varepsilon_{o}\right)^{I / 2}}}{g m_{x}^{I / 2} P_{y O}}\right],
$$

if $\mu-\varepsilon_{0} \gg T_{c}$, or

$$
\mu-\varepsilon_{\delta} \ll\left(\frac{g m_{x}^{1 / 2} P_{y 0}}{\pi d}\right)^{2}-E \lambda_{o}^{2},
$$

where $E \sim 1 \mathrm{eV}, \lambda_{o}$ is the usually normalized dimensionless interaction constant. In the opposite case, $\mu-\varepsilon_{\delta} \approx T_{c}$,

$$
T_{c}=0.0093\left(\frac{g m_{x}^{1 / 2} P_{y 0}}{d}\right)^{2} \sim E \lambda_{o}^{2} .
$$

In this extreme case $T_{c} \sim 100 \mathrm{~K}$ corresponds to $\lambda_{o} \sim 0.1$, i.e. smaller than in the usual low-temperature superconductors. However even in this case the ratio $2 \Delta T_{c}$ is 3.795 (in case $\mu-\varepsilon_{\delta} \gg T_{C}$ it has the usual value 3.52). Since the experimental value is closer to 6 , a weak coupling theory is probably insufficient for numerical predictions and can serve only for a qualitative analysis.

\section{GAP ANISOTROPY. THE E-L MODEL [14]}

The same authors as well as the Stanford group has observed another property of the photoemission spectra: the anisotropy of the energy gap $\Delta$. This was done in BSCCO - the only substance, where the ARPES technique permitted to observe a gap. Since the value at the minima fell below the resolution threshold, this was interpreted as a firm proof of the so called d-wave pairing, where the order parameter changes sign and has nodes. Actually none of these statements can be proven, since in these experiments only the magnitude of the order parameter is measured, and the resolution is finite. In case when the interaction between electrons is mediated by phonons (as this can be concluded from the isotope effect, which we will discuss later) there are no arguments in favor of d-wave pairing (such a simmetry of the order parameter appears, if the interaction is mediated by spin fluctuations or is a result of Coulomb repulsion). Therefore it can be asked whether such an anisotropy is possible within the phonon scheme.

In usual superconductors, even with an anisotropic basic energy spectrum the gap is rather isotropic (anisotropy less than 10\%). This is due to the isotropy of the interaction between electrons mediated by phonons. Even in case of a high state density in some regions of the quasi-momentum space the equation of the type 


$$
\Delta(p)=\int K\left(p, p^{\prime}\right) f\left[\Delta\left(p^{\prime}\right)\right] d^{3} p^{\prime} l(2 \pi)^{3}
$$

will connect $\Delta$ at any $p$ with the singular region, and hence will not permit $\Delta(p)$ to be anisotropic, except for the case, if $K\left(p, p^{\prime}\right)$ is anisotropic. In principle this could be achieved by spin-fluctuation exchange, if their relevant momenta were concentrated within narrow intervals around some particular values.

However we consider, as much more natural, the idea of a small momentum transfer. Although the high $T_{c}$ materials are certainly metals, they are in some sense close to ionic crystals This can be traced from the experimental observation that in the infrared as well as in Raman measurements the phonon peaks are very high (see e.g. ref. [15], [4]). It has also to be considered that structural models consisting of differently charged ions are of much help, not less than band structure calculations based on the LDA method. The cross-over from metals to ionic crystals can be understood if one imagines that the Coulomb forces, which are the basis of all real interactions, are poorly screened, i.e. the Debye screening radius is not of atomic size, as in good metals, but much larger. The expression for the square of the reciprocal Debye radius is $a^{2}=4 \pi\left(e^{2} / \varepsilon_{\infty}^{2}\right) v(\mu)$, where $\varepsilon_{\infty}$ is the part of the dielectric constant due to ionic cores, and $v(\mu)$ - the state density. Substituting eq. (12) we obtain:

$$
a^{2}(0)=\frac{2 \sqrt{2} N_{s} e^{2} m_{x}^{1 / 2} P_{y} 0}{\pi \varepsilon_{\infty} d\left(\mu-\varepsilon_{0}\right)^{1 / 2}},
$$

where $N_{\mathrm{s}}$ is the number of singular points per Brillouin zone and we have taken into account 2 spin projections.

From this formula it can be seen that $a^{2}(0)$ is small only in case of large $\varepsilon_{\infty}$. The experimental measurements of the dielectric constant as function of frequency [16] give very different values, sometimes larger than 1000 , but in case of a complicated energy spectrum it is not easy to decide how $\varepsilon_{\infty}$ has to be extracted, and so we will simply make the assumption that $\varepsilon_{\infty} \gg I$. There appear several momentum scales in the problem. For simplicity we consider the extreme case

$$
p_{F l}=\left[2 m_{x}\left(\mu-\varepsilon_{o}\right)\right]^{1 / 2} * a * P_{y 0} * d^{-1} * K,
$$

where $p_{F I}$ is the 1D Fermi momentum, $d$ is the period along $c$, and $K$ is the reciprocal lattice period in the plane; in reality other cases are also possible.

There is no unique way to chose the model interaction. We will assume that the square of the electron-phonon interaction matrix element entering the phonon mediated electron interaction, as well as the electron-phonon scattering probability, is multiplied by 


$$
\left(\frac{a^{2}}{k^{2}+a^{2}}\right)^{n} .
$$

where $k$ - the transferred momentum, $n>1$. In the BCS equation this factor will lead to the replacement, compared to the previous section:

$$
\frac{g P_{y 0}}{(2 \pi)^{2} d} \rightarrow \frac{g a^{2}}{8 \pi^{2}(n-1)} .
$$

Let us first consider the vicinity of the singularity. Substituting the modified phonon mediated interaction into the BCS equation we obtain

$$
\frac{1}{\lambda}=\frac{1}{2} \int_{-\mu_{I}}^{\infty} \frac{d \xi \mu_{1}^{1 / 2}}{\left(\xi+\mu_{1}\right)^{1 / 2}} \frac{\operatorname{th}\left[\left(\xi^{2}+\Delta_{o}^{2}\right)^{I / 2} / 2 T\right]}{\left(\xi^{2}+\Delta_{o}^{2}\right)^{1 / 2}},
$$

where $\mu_{I}=\mu-\varepsilon_{\delta} \ll \mu, \Delta_{o}$ is the large gap in the vicinity of the singularity, and

$$
\lambda=\frac{g a^{2}\left(2 m_{x}\right)^{I / 2}}{(2 \pi)^{2}(n-1) \mu_{1}^{I / 2}} .
$$

At $T=0$ we obtain from eq. (20) in the limit $\mu_{4} \geqslant 4$

$$
\frac{1}{\lambda}=\ln \frac{8 \mu_{1}}{\Delta_{o}} \text {, or } \Delta_{o}=8 \mathrm{H} \mathrm{e}^{-1 / \lambda}
$$

The order parameter not in the vicinity of the singularity is small for the following qualitative reasons. In the BCS equation (16) the integration can be split in two regions. If we integrate over the vicinity of the singularity, the density of states (per unit angle) is high but the interaction is weakened by the large momentum difference $\boldsymbol{p}^{\prime}-\boldsymbol{p}$. If, however we integrate over $\boldsymbol{p}^{\prime}$ close to $p$ the density of states is small. Assuming isotropy outside of the singular points we get the equation

$$
\frac{\Delta_{l}}{\Delta_{o}}\left(1-\lambda_{I} \frac{2 \omega_{D}}{\Delta_{I}}\right)=\frac{2(n-1) a^{2(n-1)} P_{y 0}}{p^{2 n} d}
$$

where $\Delta_{1}$ is the order parameter far from the singularity, $p$ - the distance from the singularity in the plane $\left(p_{x}, p_{y}\right)$,

$$
\lambda_{I}=\frac{g m a^{2}}{(2 \pi)^{2}(n-1) p_{o}}
$$


is the normalized interaction far from the singularity, $p_{\mathrm{O}}$ and $m$ characterize the spectrum in this region. From eq. (23) it follows that $\Delta_{l}$ decreases rapidly with $p$ and reaches eventually a value corresponding to the spectrum without the singularity. On the other hand it is always finite, until $\Delta$ is finite.

What concerns the singular region, it is not connected with external regions, and $\Delta_{b}$ as well as $T_{c}$ are defined from the equation (20). As we mentioned before, it gives the $2 \Delta_{b} / T_{c}$ ratio not very far from the BCS value. It follows that the $\mathrm{E}-\mathrm{L}$ model, considered as a weak interaction theory, cannot describe quantitatively all the properties of the HTSC. Nevertheless it can be useful for understanding the origin of various unusual phenomena in these substances.

The results of the most recent experimental determination [17] of this function is presented at Fig. 8.

Fig. 8. The latest ARPES data on the angular dependence of the : superconducting energy gap in $\mathrm{Bi}_{2} \mathrm{~S}_{2} \mathrm{CaCl}_{2} \mathrm{O}_{8+\delta}$

Apart from the smallest gap region (in sec. 7 we will show how this disagreement can be cured), it fits qualitatively to the predictions of the theory described here and definitely disagrees with the "d-wave" and "isotropic s-wave" concepts.

\section{ISOTOPE EFFECT [18]}

The isotope effect is usually described by the power $\alpha$ in the presumed dependence

$$
T_{c} \propto M^{-\alpha}
$$


where $M$ is the average ion mass (in the high- $T_{c}$ copper oxides $Q_{16}$ is usually partially substituted by $q_{8}$ ). Hence the power $\alpha$ can be defined by the relation

$$
\alpha=\frac{\partial \ln T_{c}}{\partial \ln M}
$$

The dependence of type (25) is definitely true in the simplest electronphonon interaction model, where $\alpha=1 / 2$, but in most of the real cases the connection is far more complicated. Since, however, the relative variation of the ion mass is very small, formula (26) can be taken as a rather complete characteristic of the isotope effect even in cases, when the original formula (25) is not correct, and the true dependence is far from a power law.

There exists an observation that in cases, when the composition of a layered cuprate superconductor can be altered in a regular way, and the critical temperature varies with concentration, the isotope effect depends on composition, and the lower the critical temperature, the larger is $\alpha$. The known examples are $\left(\mathrm{Y}_{1-\mathrm{x}} \mathrm{Pr}_{\mathrm{x}}\right) \mathrm{Ba}_{2} \mathrm{Cu}_{3} \mathrm{O}_{7-\delta}$ with varying $\mathrm{x}$ [19], [20], $\mathrm{YBa}_{2}\left(\mathrm{Cu}_{1-\mathrm{x}} \mathrm{Zn}_{\mathrm{x}}\right)_{3} \mathrm{O}_{7-\delta}[21], \mathrm{Y}\left(\mathrm{Ba}_{2-\mathrm{x}} \mathrm{La}_{\mathrm{x}} \mathrm{Cu}_{3} \mathrm{O}_{7}\right.$ [22] and (La2$\left.{ }_{x} \mathrm{Sr}_{x}\right)_{\mathrm{CuO}_{4}}$ as well as $\left(\mathrm{La}_{2-x} \mathrm{Ba}_{\mathrm{x}}\right) \mathrm{CuO}_{4}[23]$, where in fact a nonmonotonous dependence $\alpha(x)$ was observed.

The small value of $\alpha$ of the order of $0.019 \pm .005$ for $\mathrm{YBa}_{2} \mathrm{Cu}_{3} \mathrm{O}_{7}$ [24] has lead (and still does) to conclusions about the non-phonon mechanism of electron interaction, although it was mentioned rather early by J.Labbe and J.Bok [13] that a simple saddle point in the electron spectrum enhancing superconductivity due to an increased density of states in some region close to the Fermi level, can replace the Debye frequency as a cut-off of the interaction, by some electronic energy limit independent on the ion mass. Such an idea applies even better to the extended saddle point since the singularity in the density of states is much stronger.

In the framework of the E-L model the momentum region of the singularity in case of small angle scattering is singled out in the sense of definition of the order parameter: all the necessary information belongs only to this region. On the contrary, the order parameter in other regions is defined by its value in the singular region: it is finite until it does not vanish in the singular region. This means that the critical temperature is also defined only by the singular region, and hence we can consider only this region and not bother about the rest. In the case of an extended saddle point singularity the integral in the BCS self-consistency equation is convergent, and the corresponding energy scale is equal to

$$
\mu_{1}=\mu-\varepsilon \text {, }
$$

i.e. the Fermi energy calculated from the saddle point. This energy does not depend on the ion mass. It can happen, however, that $\mu_{y}$ exceeds some $\omega_{0}$ which is the true interaction cut-off. In this case $\omega_{0}$ becomes the integration limit despite the convergence of the integral, and hence the order parameter and the critical temperature will start to depend on the ion mass. This all happens gradually and for practical purposes it can be represented as 
variation of $\alpha$ as defined by eq. (26), since, as we mentioned, the actual . variations of $M$ are small. We will consider here the case $\mu_{Y} » T_{c}$, and at the end we will briefly discuss the case $\mu_{1} \leq T_{c}$ which corresponds to substances with the lowest $\alpha$ and highest $T_{C}$.

As before, we assume that the true interaction has the form

$$
V(k)=g\left(\frac{a^{2}}{k^{2}+a^{2}}\right)^{n} \frac{\omega^{2}(k)}{\left(\xi-\xi^{\prime}\right)^{2}-\omega^{2}(k)} \text {, }
$$

where $\omega$ is the phonon energy and $\xi, \xi^{\prime}$ - the electron energies before and after scattering. According to the previous considerations the order parameter in the region of the singularity does not depend on momenta.

Since the electron energy depends only on $p_{x}$, we can integrate the interaction (5) with respect to $p_{y}$ and $p_{z}$, and after this we obtain

$$
\int \frac{d k_{y} d k_{z}}{(2 \pi)^{3}} V(k) \rightarrow \frac{g a^{2}}{8 \pi^{2}(n-1)} \frac{\omega_{o}^{2}}{\left(\xi-\xi^{\prime}\right)^{2}-\omega_{o}^{2}}
$$

where $\omega_{b} \sim c a$ is some characteristic phonon energy at $k \sim a$. Usually the factor with $\phi_{8}$ in the interaction (29) is replaced for simplicity by

$$
\frac{\omega_{0}^{2}}{\left(\xi-\xi^{\prime}\right)^{2}-\omega_{0}^{2}} \rightarrow\left\{\begin{array}{l}
-1\left|\xi-\xi^{\prime}\right|<\omega_{0} \\
0\left|\xi-\xi^{\prime}\right|>\omega_{0}
\end{array} .\right.
$$

. Unfortunately, due to the singular density of states, which is proportional to $\left(\varepsilon-\varepsilon_{0}\right)^{-1 / 2}=\left(\xi+\mu_{\eta}\right)^{-1 / 2}$ the interaction (29) as well as its. simplified form (30) lead to unphysical singularities at $\mu_{q}=\omega_{b}$. The origin lies in the replacement of the integration over phonon frequencies by some fixed frequency $\omega_{\delta}$. On the other hand such a replacement is very helpful for simplification of the theory. Therefore, instead of (30), we will use another simplified form, which has also the property of being confined to a certain energy interval around the Fermi energy and at the same time permits to avoid nonphysical singularities in the final expressions. We will substitute the frequency dependent factor in (29) by

$$
-\frac{\omega_{o}^{2}}{\left(\xi-\xi^{\prime}\right)^{2}+\omega_{o}^{2}} \text {. }
$$


Here we are interested in $\Delta$ at the Fermi surface, and hence we put $\xi=0$. The interaction (31) enters the BCS self-consistency relation, and hence we have

$$
\frac{1}{\lambda}=\frac{1}{2} \int_{-\mu_{1}}^{\infty} \frac{d \xi \mu_{I}^{I / 2}}{\left(\xi+\mu_{1}\right)^{1 / 2}} \frac{\omega_{o}^{2}}{\omega_{o}^{2}+\xi^{2}} \frac{i h\left[\left(\xi^{2}+\Delta^{2}\right)^{I / 2}[2 T]\right.}{\left(\xi^{2}+\Delta^{2}\right)^{I / 2}},
$$

where $\lambda$ has the same meaning as before (eq. (21)).

At $T=T_{c} \Delta=0$. Performing the integrations we will assume that $T_{c} \ll \mu_{1}$, $\omega_{o}$. The result will be

$$
\begin{gathered}
\frac{1}{\lambda}=\ln \frac{8 \eta_{4}}{\pi T_{c}}-f(x), \\
x=\omega_{0} / \mu_{1}, \gamma=\mathrm{e}^{\mathrm{C}}=1.781 \text {. The asymptotic values of } f(x) \text { are } \\
f(x) \approx \begin{cases}\ln \frac{4}{x}-\frac{1}{8}\left(3 \ln \frac{4}{x}-\frac{7}{2}\right) x^{2} & x<1 \\
\frac{\pi}{2 \sqrt{2 x}} & x \geqslant 1\end{cases}
\end{gathered}
$$

From formula (33) we define $\alpha$ according to eq. (26). Assuming that $\omega_{o}$ is proportional to $M^{-1 / 2}$ we obtain: $d(\ln x)=-1 / 2 d(\ln M)$. According to . eq.(9). The asymptotic values at small and large values of $x$ are

$$
\alpha(x) \approx\left\{\begin{array}{ll}
\frac{1}{2}-\frac{1}{8}\left(5-3 \ln \frac{4}{x}\right) x^{2} & x \ll 1 \\
\frac{\pi}{8 \sqrt{2 x}} & x \approx 1
\end{array} .\right.
$$

Experimental results are always given in the form of the dependence $\alpha\left(T_{c}\right)$. Although we have obtained equations describing $T_{c}$ and $\alpha$, they include an unknown interaction. More important, the characteristic phonon frequency can be renormalized, and hence depend on the electron density of states, i.e. on $\mu_{I}$. At least, our attempts to compare the theory with the experiment on the basis of the assumption of a constant $\varphi$ have failed. Since we do not want to introduce doubtful concepts about the phonon renormalization, we will perform the comparison in a different way.

From experiments we have the connection between $T_{\mathcal{C}}$ and $\alpha$. According to our concept there should be little difference between compounds with different substitution of the constituents, provided that the $\mathrm{CuO}_{2}$ planes are left intact. This is in fact confirmed by the data for 
$\left(\mathrm{Y}_{1-x} \mathrm{Pr}_{\mathrm{x}}\right) \mathrm{Ba}_{2} \mathrm{Cu}_{3} \mathrm{O}_{7-\delta} \delta$ and $\mathrm{Y}\left(\mathrm{Ba}_{2-x} \mathrm{La}_{x}\right) \mathrm{Cu}_{3} \mathrm{O}_{7}$ (Table III in ref. [25]), and we will approximate them by a linear dependence (see Fig.9):

$$
\alpha=0.623-0.00637 T_{\mathcal{C}}(\mathrm{K}), 38.3 \mathrm{~K} \leq T_{c} \leq 92.3 \mathrm{~K}
$$

Fig. 9. Experimental data on the dependence of $\alpha$ on $T_{C}$ for $\mathrm{Y}\left(\mathrm{Ba}_{2-\mathrm{x}} \mathrm{Lax}_{\mathrm{X}} \mathrm{Cu}_{3} \mathrm{O}\right.$ [ [22] (triangles) and $\left(\mathrm{Y}_{1-\mathrm{x}} \mathrm{Pr}_{\mathrm{X}}\right)_{\mathrm{Ba}} \mathrm{Cu}_{3} \mathrm{O}$ [19],[20] (circles). The points were taken from table III of the review article by J.P.Franck [25]. The dashed straight line is the least square fit

- (eq. (36)).

This we can use to define the dependence of $\mathrm{x}$ on $T_{C}$ with the help of the equation for $\alpha(x)$. Substituting $x$ in eq. (33) we can obtain the connection between $\mu_{I}$ and $T_{c}$. This will be the prediction for future experiments, since the photoemission experiments permit to measure $\mu_{1}$ for compounds with reduced $T_{c}$, provided that single crystalline samples will be available.

For convenience we rewrite eq. (33) in the form

where

$$
\ln T_{c}+f(x)=\ln \frac{8 n_{1}}{\pi}-b \mu_{I}^{1 / 2}
$$

$$
b=\frac{1}{\lambda \mu_{1}^{1 / 2}}
$$

is the unknown constant (independent on $\mu_{\eta}$ ). The program was to define $\mu_{1}\left(T_{c}\right)$ for different values of the constant $b$ and to leave the choice to experiment. It happened, however, that the right hand side as function of $\mu_{Y}$ and the left hand side as function of $T_{c}$ are nonmonotonous and have maxima. The only possibility to obtain a continuos dependence $\mu_{1}\left(T_{c}\right)$ is to chose the constant $b$ in such a way that the two maxima have equal values (otherwise we get either a discontinuity or no solution at all). This value is

$$
b=0.137 \text {, }
$$

and the corresponding dependence $\mu_{1}\left(T_{C}\right)$ is plotted at Fig. 10 . 
Fig. 10. Predicted dependence of the location of the Fermi energy with respect to the saddle point, $\mu_{\text {, }}$, on $T_{c}$ (both quantities are measured in $\mathrm{K}$ ) [18].

This definiteness is no surprise, since the basis of these calculations is the experimental dependence $\alpha\left(T_{c}\right)$ for definite compounds.

In the previous derivation it was assumed that both, $\mu_{1}$ and $\omega_{o}$, are much larger than $T_{C}$. This happens not to be the case for the largest $T_{C}$ ( $\mu_{1}=T_{c}$ is presented by the dashed line at Fig.10). Of course this can be corrected (actually already for $\mathrm{T}_{\mathrm{c}}=90 \mathrm{~K}$ the correction is small), however this region is suspicious in the sense that the small value of the isotope shift is likely not entirely defined by the cut-off of the integrals, and the phonon frequency can enter the interaction, reducing the value of $\alpha$. Otherwise small values of $\alpha$ require large values of $x$ (see eq.(35)), and hence unphysically large values of $\omega_{0} ;$; see Fig.11 representing the dependence $\omega_{0}\left(T_{c}\right)$ obtained from $x\left(T_{c}\right), \mu_{1}\left(T_{c}\right)$ and $x=\omega_{0} / \mu_{1}$.

Fig. 11. The variation of the effective phonon frequency of with $T_{c}$ (both in $\mathrm{K})$ [18].

The fact that the isotope shift for $\mathrm{Cu}^{63}-\mathrm{Cu}^{65}$ is negative and increases in magnitude with increasing $T_{C}$ (see ref. [26]), can also be considered as evidence for inapplicability of the theory, based on the assumption of the purely cut-off nature of the isotope shift, to substances with extreme $T_{c}$. On 
the other hand for the lower $T_{\mathrm{c}}$ substances the values of $\omega_{O}$ are reasonable, . and their variation can be ascribed to renormalization.

The predictions of the present theory are rather definite, but in order to check them direct determinations of the function $\alpha(x)$ have to be performed.

This is possible, in principle, by measuring $\alpha$ and 4 (by ARPES), using the same samples.

\section{RESISTIVITY IN THE NORMAL STATE [14]}

The linear temperature dependence of the normal state resistivity of high- $T_{c}$ cuprates was always a puzzle for theorists, and one of the checks for the theory to be correct was this dependence. Unfortunately, people always managed to get the linear dependence in their theories based on completely different assumptions, e.g. RVB (Anderson and Zou) [27], nested Fermi surfaces (Virosztek and Ruvalds) [28], spin fluctuations (Morita et al.) [29], oxigen chains (Abrikosov and Falkovsky) [30]. Therefore such a result can neither prove nor disprove a theory. The easiest way to obtain a linear temperature dependence is an assumption that the electrons interact mainly with some optical mode having a low frequency, and the $T$ comes from the Bose distribution $\left[\exp \left(\omega_{o} / T\right)-1\right]^{-1}$ at $T * \omega_{o}$. This sort of explanations is likely to be wrong because the high frequency resistivity $\sigma \omega)^{-1}$ at $\omega » T$ varies linearly with $\omega$ (see e.g. ref. [32]), i.e. $\omega$ replaces $T$; this can happen only if $T, \omega * \omega_{\mathcal{O}}$ - the limiting energy of quasiparticles mediating the interaction.

We are not going to make an exception and will also obtain the linear resistivity in the framework of the theory presented above. We will show that scattering of electrons from electrons at low temperatures is much larger than scattering from phonons; therefore we will consider it first (the same was true for the model of nested Fermi surfaces [28]). In the previous section we have assumed that the interaction between electrons due to exchange of phonons is stronger than the Coulomb repulsion (actually they are of the same order of magnitude). Here however the situation can be different. The matrix element of the Coulomb interaction is

$$
\frac{4 \pi e^{2} / \varepsilon_{\infty}}{k^{2}+a^{2}},
$$

whereas the interaction via phonons is

$$
g\left(\frac{a^{2}}{k^{2}+a^{2}}\right)^{n} \frac{\omega(k)^{2}}{(\delta \xi)^{2}-\omega(k)^{2}} .
$$

In the forthcoming the integral over $k$ will require $k \sim a$, and since $\delta \xi$ will be of the order of $T$, the second matrix element will be much less than the 
first one in the case $T » \omega_{O}=\omega(\alpha)$. If on the contrary $T$ « $\omega_{0}$, the second factor in eq. (16) becomes -1 , the same as in the gap equation of the previous section. Then the interaction acquires the same form as a pure Coulomb (40) with the replacement $4 \pi e / \varepsilon_{\infty} \rightarrow-g c$. The sign is of no importance, since only the square of the interaction enters the scattering probability, and the order of magnitude of both interactions is likely to be the same. Therefore we will write the interaction in the form (40) with the possible replacement $4 \pi e^{2} / \varepsilon_{\infty} \rightarrow-g a e^{2}$ in case $T \ll \omega_{0}$. We obtain

$$
\begin{aligned}
& \frac{1}{\tau_{e e}}=2 \pi\left(\frac{4 \pi e^{2}}{\varepsilon_{\infty}}\right)^{2} \\
& \quad \times \int \frac{n_{2}\left(I-n_{1}\right)\left(I-n_{2}\right) \delta\left(\varepsilon_{1}+\varepsilon_{2}-\varepsilon_{1}-\varepsilon_{2}\right)(I-\cos \theta)}{\left[\left(p_{1}-p_{1}\right)^{2}+a^{2}\right]^{2}} \frac{d_{1}^{3} d^{3} p_{2}}{(2 \pi)^{6}}
\end{aligned}
$$

where $\theta$ is the angle between the velocities before and after scattering, and $\mathbf{n}_{\mathbf{i}}$ are the Fermi functions. Integrating we obtain the final expression.

$$
\frac{1}{\tau_{e e}}=\frac{1}{2 \pi} \frac{e^{4} P_{y 0}}{\varepsilon_{\infty}^{2} d} \frac{m_{x}}{a^{2}} \frac{T}{\mu-\varepsilon_{o}}=\frac{1}{4 \sqrt{2} N_{s}} \frac{e^{2} m_{x}^{I / 2}}{\varepsilon_{\infty}\left(\mu-\varepsilon_{0}\right)^{l / 2}} T
$$

(here we have substituted the formula (17) for $\alpha^{2}$ ).

The reason why we get in this case a linear $T$ dependence instead of the usual quadratic one is that in a $3 D$ or $2 D$ dimensional case the $\delta$-function fixes the angle between the momenta whereas in the $1 \mathrm{D}$ situation there is no angle to fix, and the $\delta$-function reduces two momentum integrations to one. The coefficient in the linear dependence (43) would be large $\left(e^{2} / v_{F} \sim 1\right.$ for an ordinary metal, and here $v_{F}$ is much smaller) if not for $\varepsilon_{\infty} \Downarrow 1$. Therefore this requirement is actually the condition for the Landau Fermiliquid theory to be applicable to the layered cuprates under consideration.

Now we calculate the scattering probability from phonons; it is given by the expression

$$
\frac{1}{\tau_{e p}}=2 \pi g \int \frac{\alpha^{2 n} \omega(k) \delta[\varepsilon(p)-\varepsilon(p-k)](1-\cos \theta) d^{3} k /(2 \pi)^{3}}{\left(k^{2}+\alpha^{2}\right)^{n}\{\exp [\omega(k) / T]-1\}} .
$$


For $T » \omega_{O}$

$$
\frac{1}{\tau_{e p}}=\frac{g a^{2 n} m_{x}}{\left.\pi / p_{x}\right\rfloor} \frac{c k^{2} d k}{\left(k^{2}+a^{2}\right)^{n}[\exp (c k / T)-1]} \sim \frac{g a^{2} m_{x}}{\left.\mid p_{x}\right\rfloor} T .
$$

For $T \ll \omega_{O}$, the result is

$$
\frac{1}{\tau_{e p}}=\frac{g m_{x}}{\pi / p_{x} /} \int_{0}^{\infty} \frac{c k^{2} d k}{[\exp (c k / T)-I]}=\frac{2 \zeta(3) g m_{x} T^{3}}{\pi c^{2} / p_{x} l} .
$$

The ratio of the probabilities for $T * \omega_{8}$ has the order of magnitude

$$
+\frac{\tau_{e p}^{-1}}{\tau_{e e}^{-1}}-\frac{g e^{2} \varepsilon_{\infty}}{e^{2}} \times 1
$$

However for $T * \omega_{o}$ the electron-electron scattering is dominant. The dominance of the electron-electron scattering over the electron-phonon scattering can be concluded also from the experimental fact that with decreasing temperature below $T_{c}$ the lifetime of quasiparticles obtained from infrared measurements starts to increase rapidly [32]. If such a dominance takes place below $T_{c}$, it must definitely continue in some temperature range above $T_{c}$ :

If the singular points are at all the boundaries of the Brillouin zone, the resistivity in the ab-plane is

$$
\rho=\frac{\pi}{8} \frac{m_{x} d}{P_{y 0}\left(\mu-\varepsilon_{\delta}\right)} \frac{T}{\varepsilon_{\infty}}
$$

The crucial idea for all results obtained in the foregoing is the assumption $\varepsilon_{\infty} \gg 1$. Apart from that we have presumed a modification of the electron-phonon interaction (square of the matrix element) which is described by formula (19). We realize that this treatment is not complete. The Coulomb repulsion has not been seriously considered. In the model of strongly compressed matter [33], it compensates almost entirely the phonon attraction due to longitudinal phonons (in this case $n=1$ ); we hope that this analogy cannot be extended to such extremely anisotropic substances, as layered cuprates.

The quasi 1D spectrum appearing as a result of the "extended saddle point singularities" puts also questions about the applicability of the Fermi liquid approach, since the purely ID interacting Fermi system is more likely to be a "Luttinger liquid". We hope that the non-1D features will be sufficient to suppress the logs leading eventually to the breakdown of the 
Fermi-liquid; this all, however, has to be checked. On the other hand the quasi 10 situation may be more favorable for the "preformed pairs" idea (see e.g. ref. [34]).

\section{ON THE NATURE OF THE ORDER PARAMETER [35]}

One of the hottest topics in the theory of high- $T_{C}$ cuprates is the symmetry of the order parameter. One point of view is that the pairing is of "s-type", possibly anisotropic. According to this hypothesis the order parameter does not change its sign along the Fermi surface, and the energy gap has no nodes. This is confirmed by several experiments which definitely demonstrate a finite energy gap, e.g. the Knight shift [36], tunneling conductance in BiSCCO [37] and HgBCCO [38]. The most convincing argument in favor of this point of view is the strong isotope shift of the critical temperature in YBCO with a partial substitution of constituents $(\mathrm{Y} \rightarrow \mathrm{Pr}$, or $\mathrm{Ba} \rightarrow \mathrm{La}$ ), which we discussed before. This is a clear evidence of a phonon mechanism of superconductivity which leads to an order parameter with no nodes. One of the examples is the model described in the previous sections.

On the other hand there exists also strong evidence in favor of the so called " $d$-wave" pairing. These are the linear temperature dependence of the penetration depth at low temperatures [39] the Josephson experiments on single crystals [40] and rings, consisting of several grains [41]. One must have in mind, however, that these experiments demonstrate actually only the fact that the order parameter, as function of momentum changes sign and has nodes but do not exclude dependencies differing from the form $\Delta(k)=$ $\cos k_{x}-\cos k_{y}$ which is usually advocated by the proponents of the dwave hypothesis. Recent direct measurements of the angular dependence of the energy gap by photoemission (J.-C. Campuzano et al., see Fig. 8) did not confirm this form. The only result, which favors it, is the absence of the Josephson effect in a BiSCCO-Pb tunnel junction (surface 1 c) [42] but this result, which could mean that the integral of $\Delta(k)$ over the whole Fermi surface vanishes, is in contradiction with the observation of the Josephson effect in the same geometry with YBCO instead of BiSCCO and with the gap measurements already mentioned.

Our goal is to demonstrate that most of the observations can be explained by a very simple idea which is a development of the E-L model. Compared to it we introduce the following change. We will assume that in addition to the phonon attraction considered in sec. 4 there exists a small and short ranged repulsive interaction $U=$ const. which can represent either some part of the Hubbard repulsion at the copper sites, or the interaction mediated by spin fluctuations (taken alone, such an interaction would lead to d-wave pairing). As in sec. 4, we will presume the following inequalities:

$$
p_{F I} \ll \propto P_{O y} \ll 11 d \ll K,
$$

As shown in sec. 4 , under these conditions $\Delta$ is constant in the singular region. Substituting $V(k)+U$ into the $\mathrm{BCS}$ self-consistency equation, 
assuming the density of states and $\Delta$ in the singular region to be much larger than beyond it and integrating over $k_{y}$ and $k_{z}$ we obtain

$$
l=\frac{\lambda}{2} \int_{-\mu_{1}}^{\infty} \frac{d \xi \mu_{1}^{1 / 2}}{\left(\xi+\mu_{1}\right)^{1 / 2}} \frac{\operatorname{th}\left[\left(\xi^{2}+\Delta_{o}^{2}\right)^{I / 2} / 2 T\right]}{\left(\xi^{2}+\Delta_{o}^{2}\right)^{1 / 2}},
$$

where $\delta$ is the value of $\Delta$ in the singular region, and

$$
\lambda=\frac{\left(2 m_{x}\right)^{1 / 2}}{(2 \pi)^{2} \mu_{1}^{1 / 2}}\left(\frac{g a^{2}}{n-1}-\frac{2 U P_{0 y}}{d}\right) .
$$

We will assume the second term in the brackets to be much less than the first one, and neglect it. The solution of eq. (49) at $T=0$ in the limit $\Delta * \mu_{1}$ is

$$
\Delta=8 \mu_{q} e^{-1 / \lambda} .
$$

Now let us consider some point at the Fermi-surface, distant from the singularity. For simplicity we consider a circular Fermi surface (there is no dependence on $k_{z}$ ), and $\varphi$ will be the angular distance from one of the singular "points" (the extension of the singularity, $P_{\mathrm{Oy}}$, and the $z$-size of the Brillouin zone, $2 \pi / d$, are assumed to be small compared to the radius of the cylindrical Fermi surface; $p_{o}$, which is of the order of $K$ ). The integral in the BCS equation consists of two parts: along the singularity and beyond it. Since the density of states in the singular region as well as the value of $\Delta=\Delta_{o}$ are large, we will assume that this part of the integral dominates (estimate of the other part's contribution see below), and hence $\Delta(\varphi)$ beyond the singularity will be defined by its value in the singular region. The integral over $\xi$ will be the same, as in eq. (49), and we can replace it using this equation. Eventually we obtain the equation

$$
\frac{\Delta(\varphi)}{\Delta_{o}}=\frac{2(n-1) P_{0 y}}{a^{2} d}\left\{\left(\frac{x}{2 \mathrm{p}}\right)^{2 \mathrm{n}}\left[\sin ^{-2 \mathrm{n}}\left(\frac{\varphi}{2}\right)+\sin ^{-2 \mathrm{n}}\left(\frac{\varphi-\varphi_{0}}{2}\right)\right]-\frac{\mathrm{U}}{\mathrm{g}}\right\}
$$

where $p_{o}$ is the Fermi momentum, and $\varphi_{o}$ - the location of the next singularity (in general a sum over locations of all singularities has to be taken). This formula describes the behavior of $\Delta(\varphi)$ far from the singularities, i.e. at not too small values of $\varphi$. For description at any angle a simple interpolation 


$$
\sin ^{2} \varphi / 2 \rightarrow \sin ^{2} \varphi / 2+\text { const }
$$

with the const chosen so that $\Delta(0)=\Delta_{o}$, can be used. The minimal value of the first term in the curly brackets of eq. (7) is at $\varphi=\varphi_{o} / 2$, and its value is $2\left[a /\left(2 p_{O} \sin \left(\varphi_{O} / 4\right)\right)\right]^{2 n}$. If this is smaller than $U / g$, then $\Delta(\varphi)$ has a negative value somewhere between the maxima, and hence the gap has two nodes in this region. In case if $\varphi_{O}=\pi / 2$, as it happens in BiSCCO, the nodes have to be located symmetrically around $\pi / 4$, and this corresponds to the observations of J.-C. Campuzano et al., which are presented at Fig. 8. If $\varphi_{O}=\pi$, which is most likely to be the case in $\mathrm{YBCO}$, the negative values of $\Delta$ are located around $\pi / 2$, i.e. if the positive maxima correspond to the adirection, the negative values are around the $b$-direction, which is exactly what is seen in experiments measuring the phase of the order parameter.

The necessary conditions for all that to be true is a sufficiently small value of $U$ :

$$
U \ll g \alpha^{2} d / P_{0 y}
$$

If this condition is fulfilled, $\Delta$ in the singular region will be defined selfconsistently by eq. (49), and hence the critical temperature will be also defined by this equation. As shown in sec. 5 , in case, when the characteristic phonon frequency $\omega_{0} \leq \mu_{l}$ ( $\omega_{0}$ is an optical frequency, or acoustical frequency at $k=a$ ), the integration in eq.(49) has to be cut off at $\omega_{o}$, and a regular isotope effect appears. Since this is observed in experiment, we believe that the condition (53) is reasonable. Another concern could be the part of the integral in the equation for $\Delta(\varphi)$ outside the singular region. With respect to the terms, which we have kept, it is either of the order of $p_{F 1} / p_{o}$, or $\left[U P_{0 y} /\left(g a^{2} d \times\left(p_{F 1} / P_{0 y}\right)\right.\right.$. Both quantities are small.

Since there is no reason for the integral $\int \Delta(\varphi) d \varphi$ to vanish, there should be a Josephson current in the HTSC-Pb junction if the boundary is normal to the c-axis, although it may be smaller than what could be expected from an estimate based on $\phi$. The failure to observe it in a BiSCCO-Pb contact could be due to the weak hopping between the $\mathrm{CuO}_{2}$ layers. The Fermi surface in this case is an almost straight cylinder, and the c-component of the electron velocity is very small. If the electron crosses the barrier keeping the direction of its velocity, it has to go a very long path. This decreases drastically the tunneling probability and can desroy the Josephson current. This does not happen if the boundary is parallel to the c-axis, because then the velocity normal to the boundary is large, and also in YBCO, where due to the chains the hopping between the $\mathrm{CuO}_{2}$ layers is stronger, and hence the c-component of the velocity is much larger. In these cases the Josephson effect was really observed [42], [43]. 
The idea presented above solves also an important problem about the suppression of superconductivity by nonmagnetic impurities. In case of dwave pairing one would expect the necessary condition to be $\tau \Delta \leq 1$, whereas in case of $\mathrm{s}$-wave pairing it could happen only at $\tau \dot{F} \leq 1$. This criterion is rather difficult to apply, because in the HTSC the ratio $\Delta \varepsilon_{F}$ is not so small as in low-temperature superconductors, and it is also not very clear which impurities behave as nonmagnetic. Nevertheless the general opinion is more inclined to interpret experimental data in terms of the condition $\tau \varepsilon_{F} \leq I$ (A.Leggett, concluding remarks at the Argonne Workshop, June 1994). In our scheme it would be rather natural, since the impurities

are most likely ionized, the interaction of electrons with them is weakly screened and long ranged, and it would not mix the singular and remote regions of momentum space. This, however, has to be checked.

There is also a question about the tunneling conductance. Experiments on BiSCCO [37], and $\mathrm{HgBCCO}$ [38] show a small conductance at $\mathrm{eV}$ less than some large gap with $2 \Delta(0) / T_{C}-6-8$ (see Fig. 12). This seems in contradiction with the present results, as well as with the d-wave and anisotropic s-wave concepts. Our scheme can explain the tunneling results as follows. The tunneling conductance is proportional to the density of states. In the nonsingular regions not only the gap, but also the density of states (per unit solid angle), is much lower than in the singular-region. Therefore it contributes only a small background; only, when $\mathrm{eV}=\Delta$ is

Fig. 12. Tunneling conductance of $\mathrm{HgBa}_{2} \mathrm{CuO}_{4}+\delta$ (niobium tip) [38]

reached, the conductance becomes large (the background is usually attributed to normal inclusions).

The model, presented here, cannot explain all the data. Even with an energy dependent one-dimensional density of states the maximal value of $2 \Delta(0) / T_{C}$, which can be obtained from eq. (49), is less than 4 (see sec. 3), whereas the experimental values are around 6 to 8 . This contradiction can be due to the fact that we apply the simple BCS-type theory, whereas the increased density of states makes the effective interaction strong. This has to be resolved in future studies. 


\section{ACKNOWLEDGMENT}

The author would like to express his gratitude to many colleagues for stimulating discussions, and particularly to J.-C. Campuzano, K.Gofron, and J.P.Franck, who communicated many of their data prior to publication.

This work was supported by the Department of Energy under the contracts \# W-31-109-ENG-38.

\section{REFERENCES}

[1] A.A.Abrikosov (1991), Physica C 182, 191

[2] A.A.Abrikosov, L.A.Falkovsky (1961) Sov.Phys. JETP 13, 179

[3] A.A.Abrikosov, L.A.Falkovsky (1988) Physica C 156, 1

[4] M.Boekholt, M.Hoffmann, G.Guentherodt (1991) Physica C 175, 42

[5] M.Tachiki et al (1990) Z.Phys. B Cond. Matt. 80, 161

[6] A.Abrikosov (1992) Physica C 201, 413

[7] T. Hasegawa et al (1992) J. Phys. Chem. Solids 53, 1643

[8] A.A.Abrikosov (1988) Fundamentals of the Theory of Metals, NorthHolland, Amsterdam,

[9] A.A.Abrikosov, R.A.Klemm (1992) Physica C 191, 224

[10] A.A.Abrikosov, J.-C. Campuzano, K.Gofron (1993) Physica C 214, 73

[11] Z.-X. Shen et al. (1993) Phys. Rev. Lett. 70, 1553

[12] D.L.Novikov, A.J.Freeman (1993) Physica C 212, 233; 216, 273

[13] J.Labbe, J.Bok (1987) Europhys. Lett. 3, 1225

[14] A.A.Abrikosov (1994)Physica C 222, 191

[15] A.M.Pao et al. (1990) Phys. Rev. B 42, 193

[16] G.P.Mazzara et al. (1993) Phys. Rev. B 47, 8119; G.Cao et al. (1993) Phys. Rev. B 47, 11510

[17] J.-C. Campuzano et al., unpublished

[18] A.A.Abrikosov, submitted to Physica $C$

[19] J.P.Franck et al. (1991) Physica $B \quad 169,697$

[20] J.P.Franck et al.(1991) Phys. Rev, B 44, 5318

[21] J.P.Franck et al. (1991) Physica $C$ 185-189, 1379

[22] H.J.Bornemann a. D.E.Morris (1991) Phys. Rev. B 44, 5322

[23] M.K.Crawford et al.(1990) Phys. Rev. $B$ 41, 182

[24] D.E.Morris et al. (1988) Phys. Rev. B 37, 5936

[25] J.P.Franck (1994)Experimental Studies of the Isotope Effect in High

Temperature Superconductors; in the Series "Physical Properties of

High $\mathrm{T}_{\mathrm{C}}$ Superconductors", Ed. D.Ginsberg, Vol. IV

[26] J.P.Franck a.D.D.Lawrie (1994) Bull. Am. Phys. Soc. 39(1), Program of the 1994 March Meeting, S8 1, p.914

[27] P.W.Anderson, Z.Zou (1988) Phys. Rev. Lett., 60, 132

[28] A.Virosztek and J.Ruvalds (1990) Phys. Rev. B 42, 4064

[29] T.Morita, Y.Takahashi and K.Ueda (1990) Journ. Phys. Soc. Japan 59,2905

[30] A.A.Abrikosov and L.A.Falkovsky (1990) Physica C 168, 556

[31] Y.Watanabe et al. (1989) Phys. Rev. B 40, 6884; R.T.Collins et al., (1989) Phys. Rev. B 39,6571; R.T.Collins et al. (1990) Phis. Rev. Lett. 64, 84; J.H.Kim et al. (1990) Phys. Rev. B 41, 7251 
[32] D.A.Bonn et al. (1992) Phys. Rev. Lett. 68, 2390

[33] A.A.Abrikosov (1961) Sov. Phys. JETP 12, 1254; (1962) Sov. Phys. JETP 14, 408

[34] M.Randeria et al. (1992) Phys Rev Lett . 69, 2001; C. Sa de Melo, M.

Randeria and J. Engelbrecht (1993) Phys. Rev. Lett. 71, 3202

[35] A.Abrikosov (1994) submitted to Phys. Rev. Lett.

[36] S.E.Barrett et al. (1990) Phys. Rev. B 41, 6283; M.Takigawa et al.

(1989)Phys. Rev. B 39, 7371

[37] Ch.Renner et al. (1994) Physica B 194, 1689

[38] Jun Chen et al., unpublished

[39] W.N.Hardy (1993) Phys. Rev. Lett. 70, 3999; D.A.Bonn et al.

(1993) Phys. Rev. B 47, 11314

[40] D.A.Wollmann et al. (1993) Phys. Rev. Lett. 71, 2134

[41] C.C.Tsuei et al., preprint

[42] H.Z.Durusoy et al., preprint

[43] A.G.Sun et al. (1994) Phys. Rev. Lett. 72, 2267 


\section{Figures}

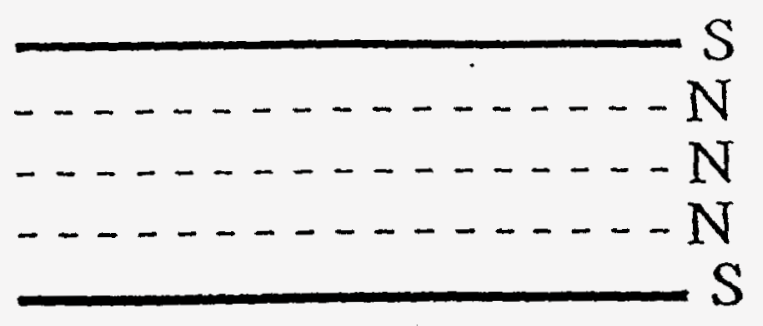

Tachiki, Takahashi, Steglich, Adrian, 1990
(2)

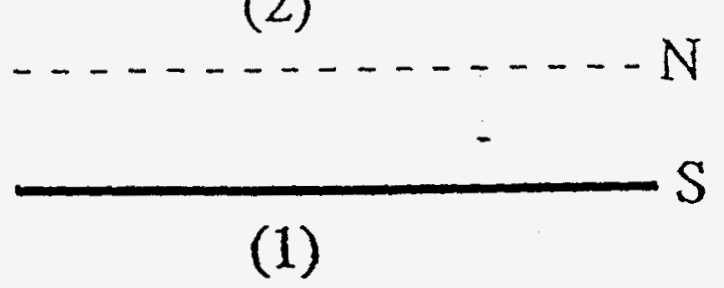

present model

$\beta=m_{1} / m_{2}<1, t \ddot{<}<\Delta$ 


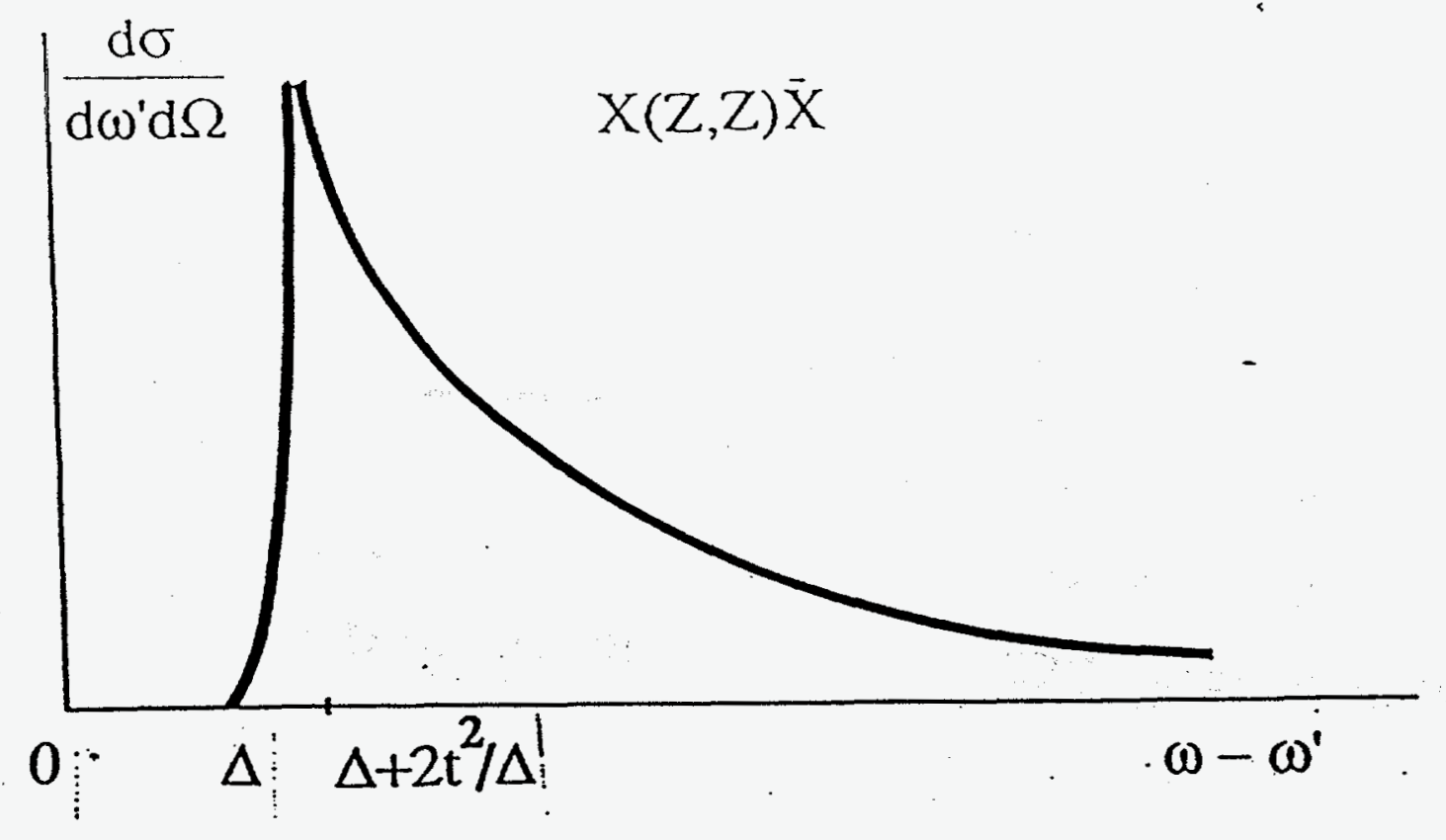

fig. 2 


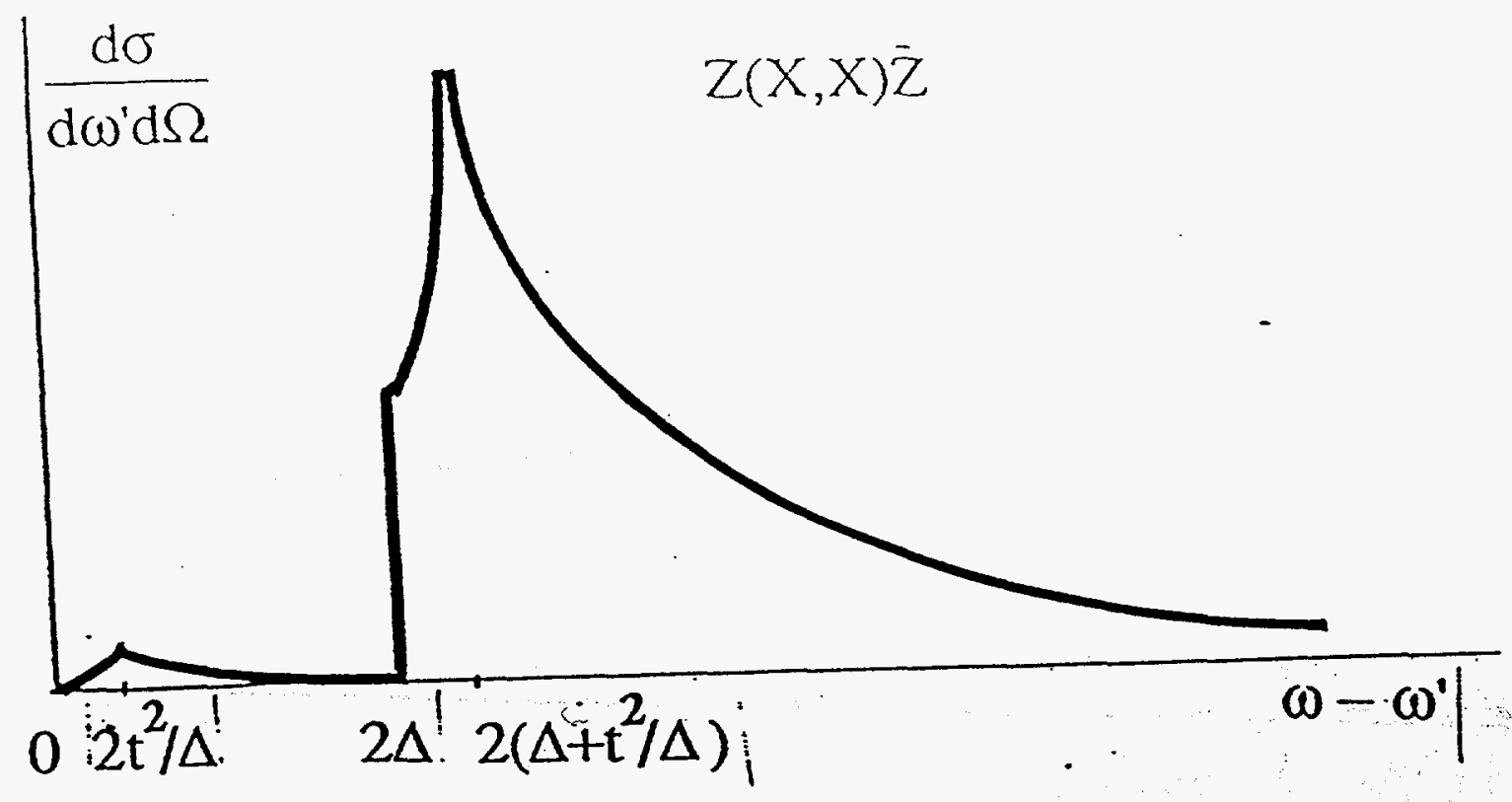




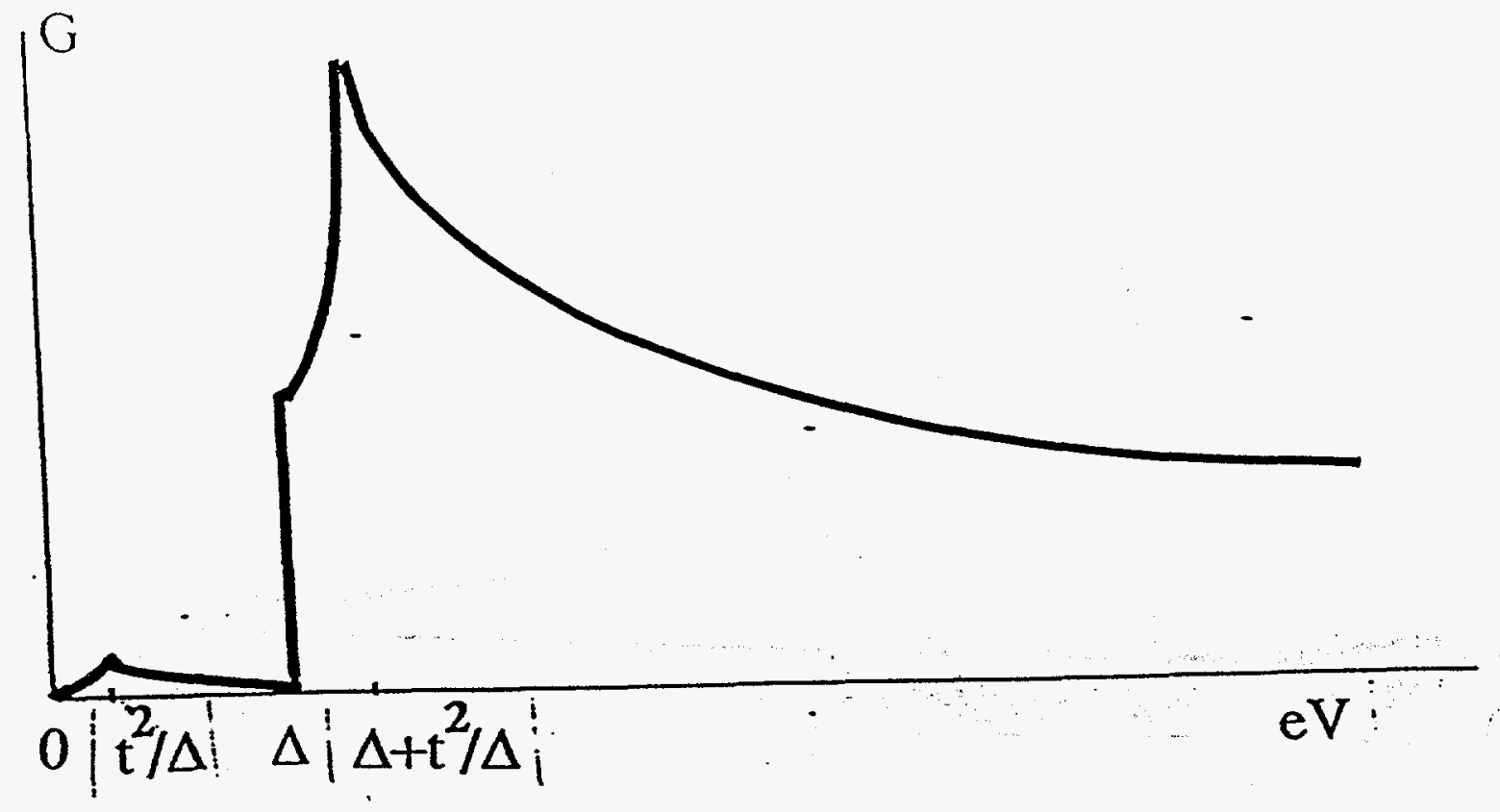

fig. 4 


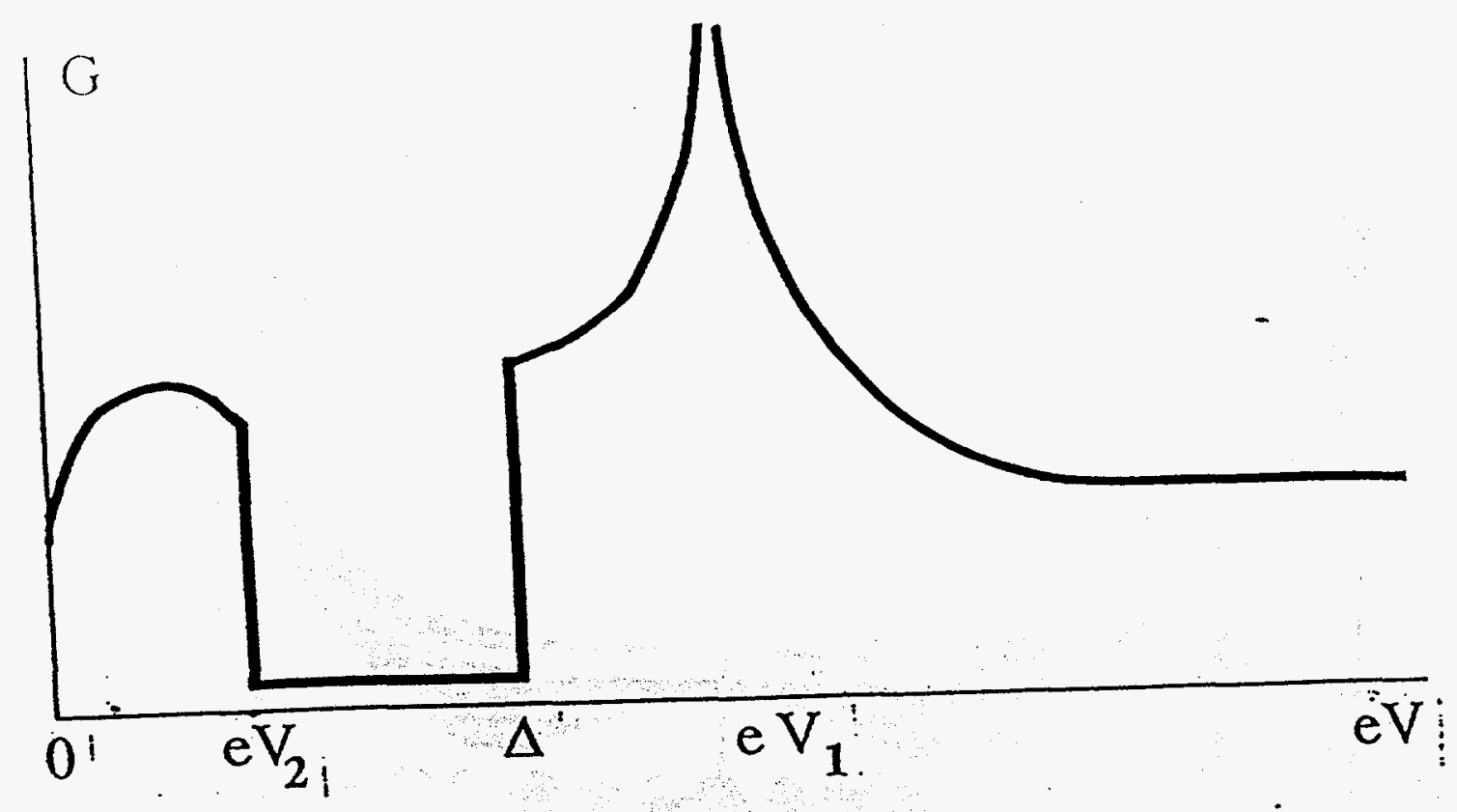


Y124 meannementes

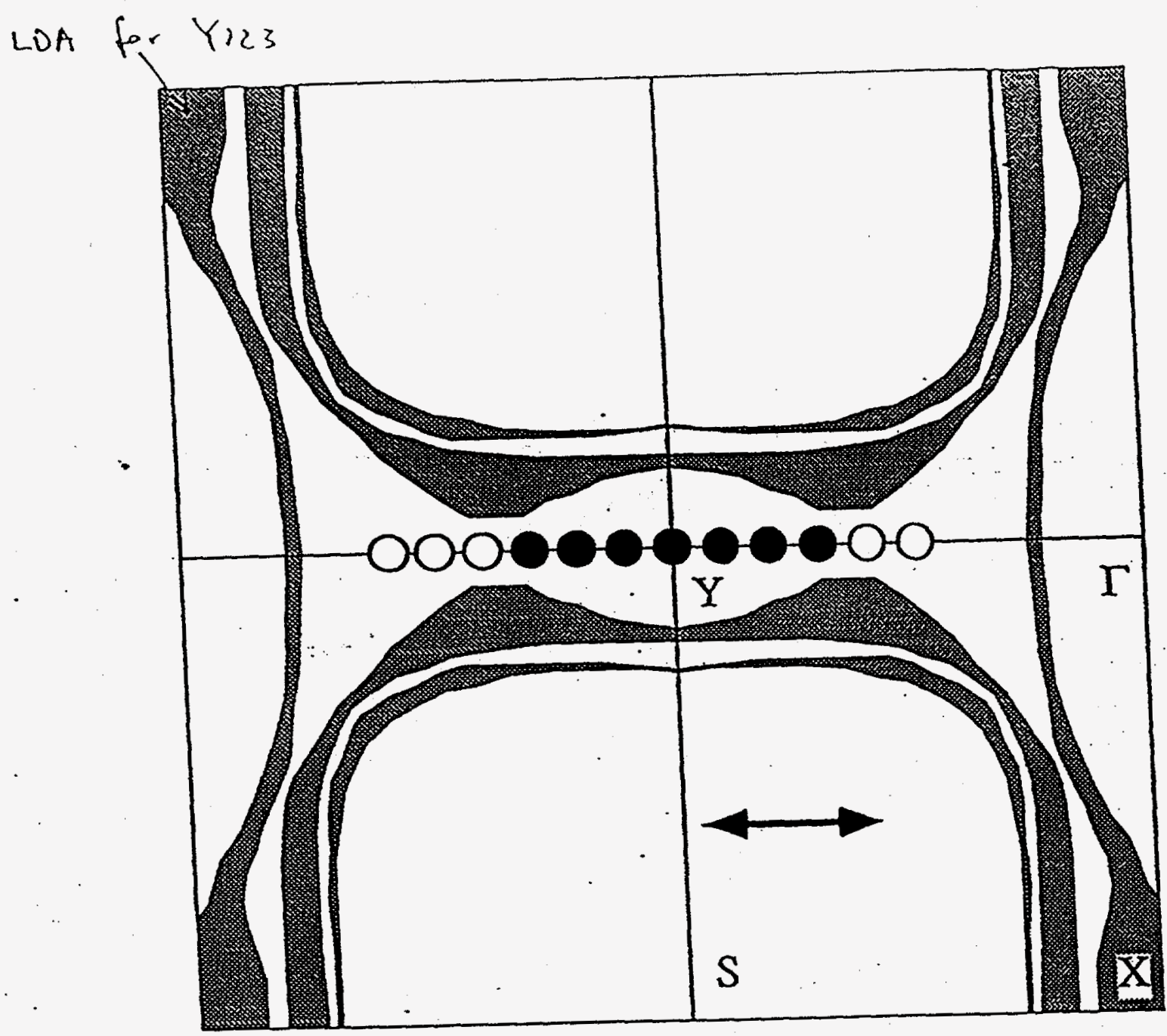

- Band is flat at $\sim 20$ med - Band has downward curvature

fig. 6 


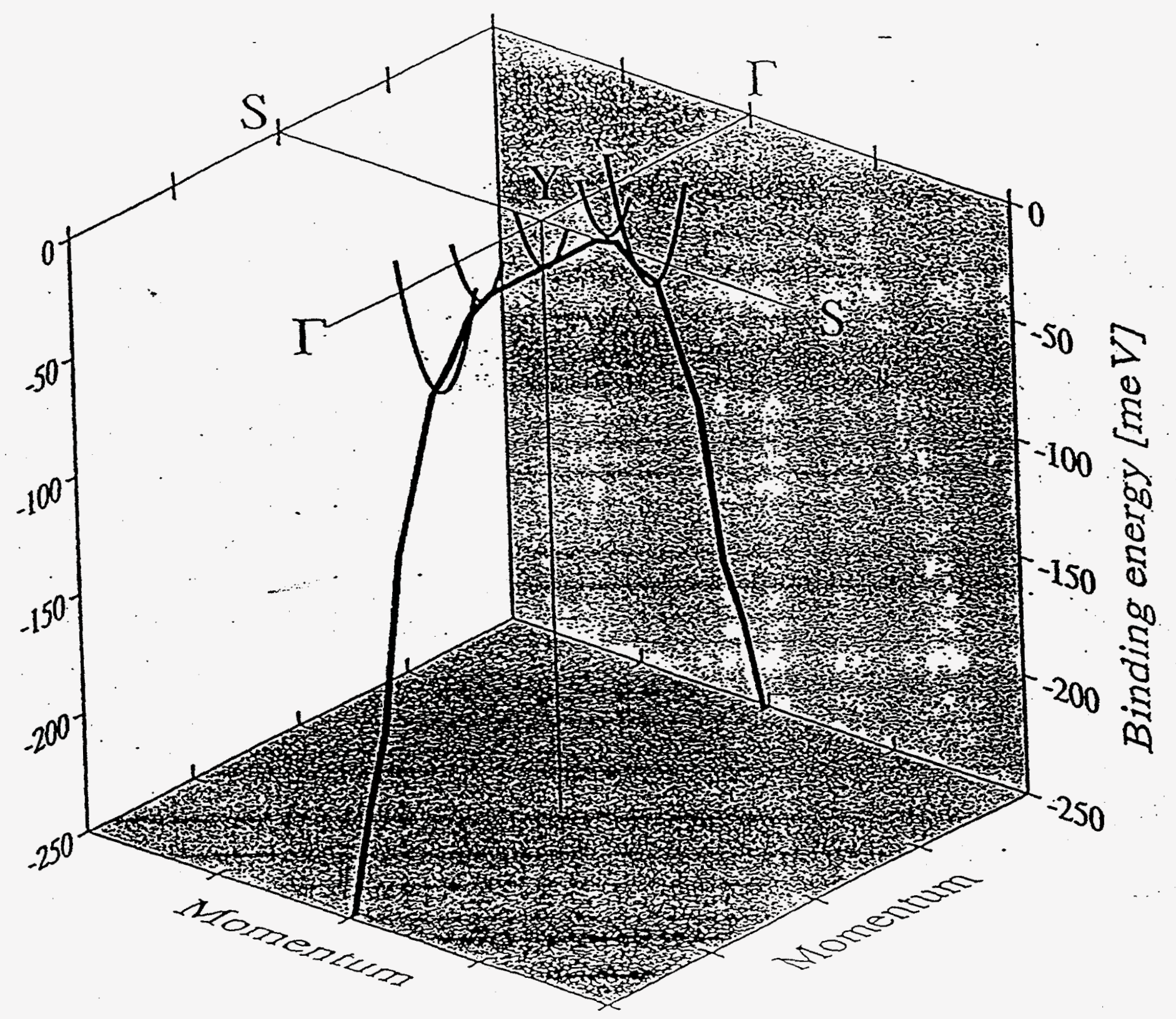

fig.? 


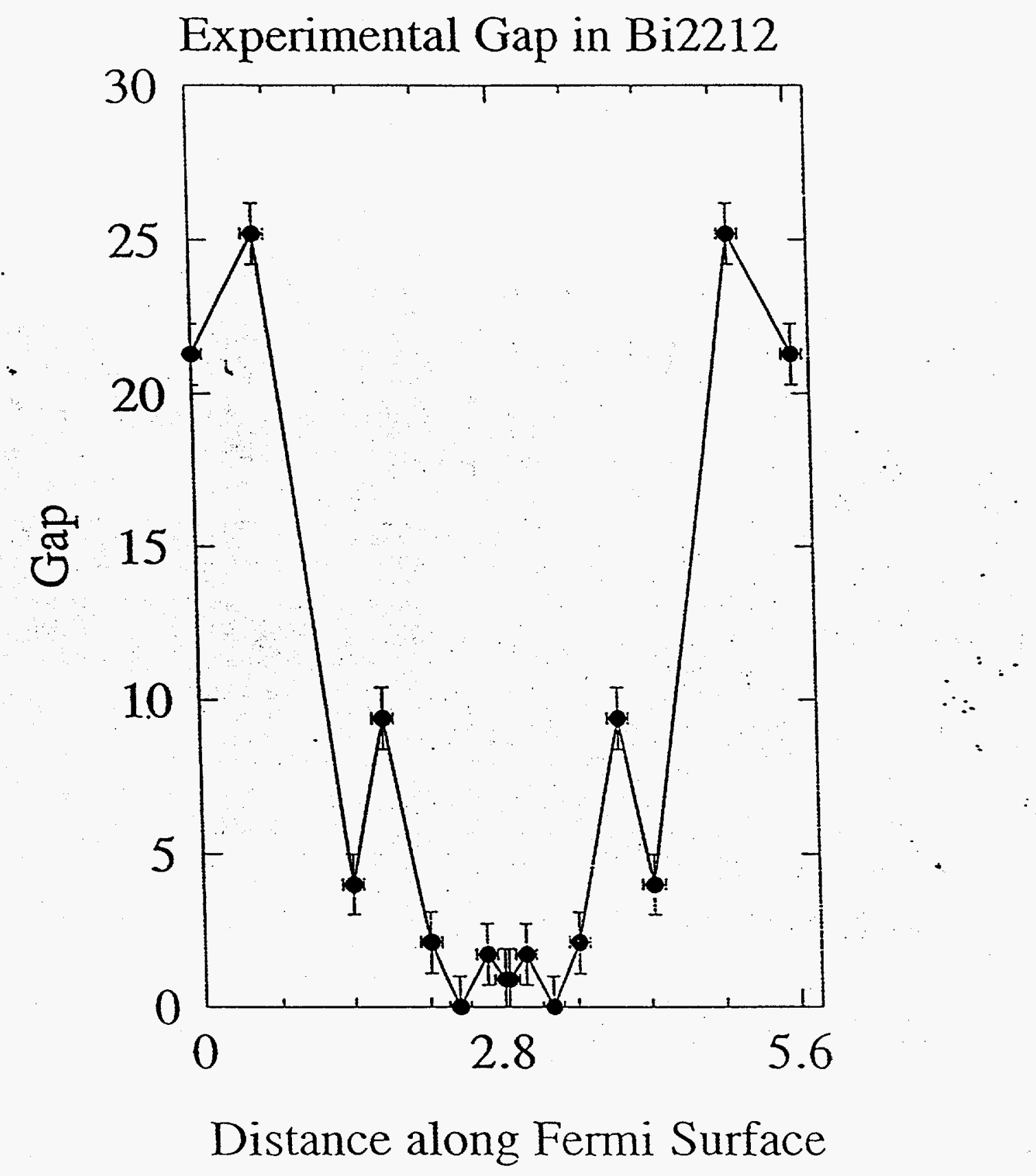

fig. 8 


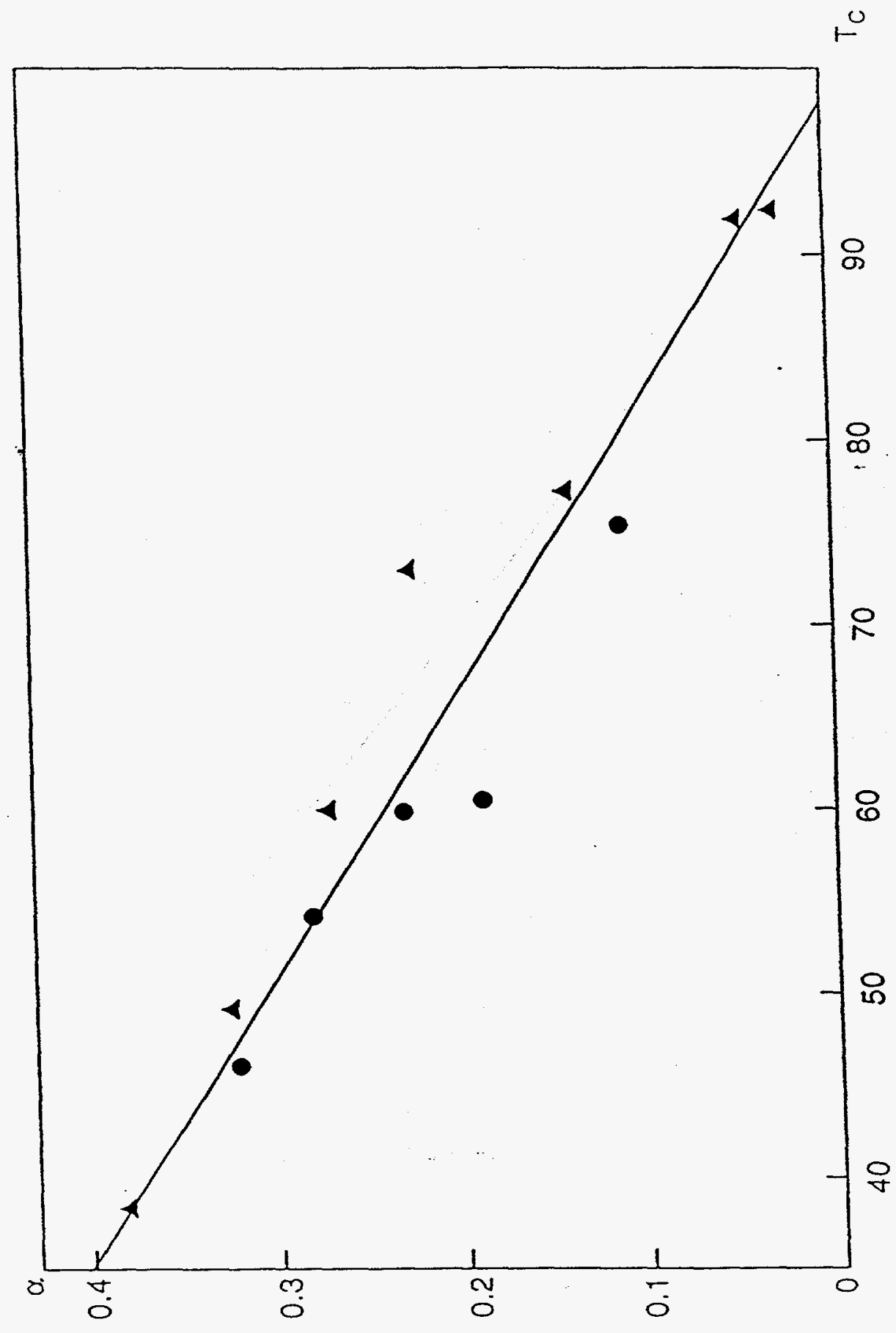




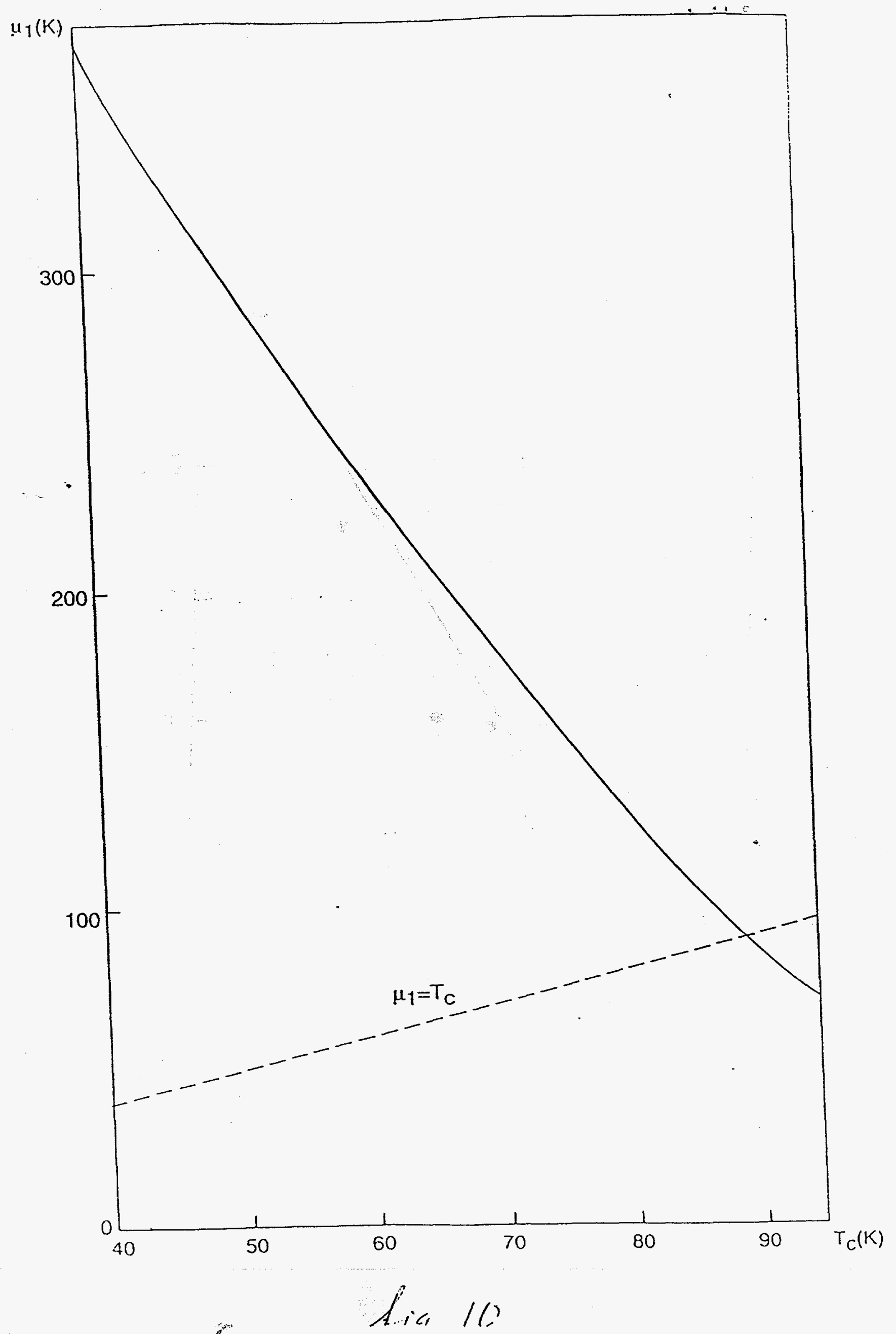




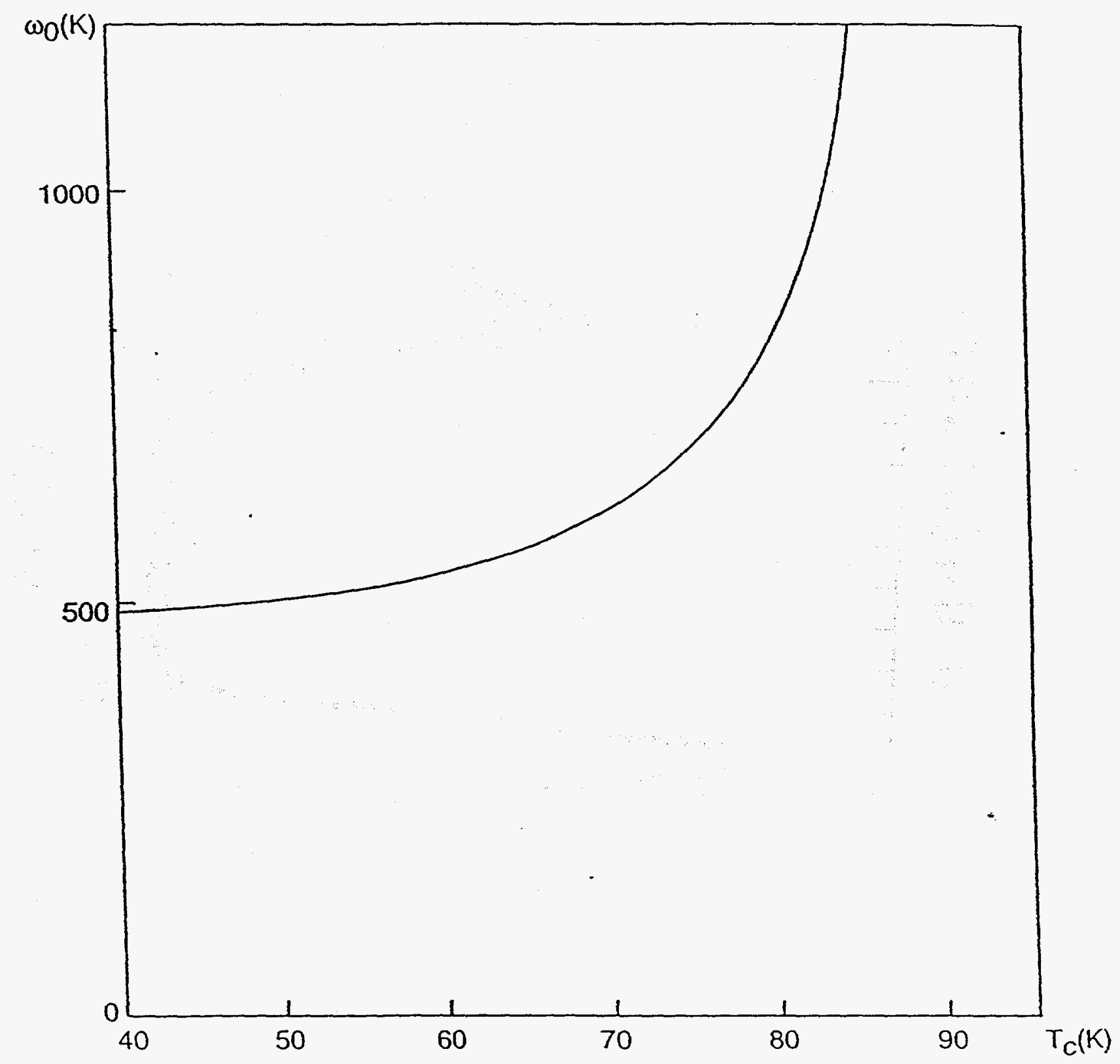

- $f_{i q} .11$ 


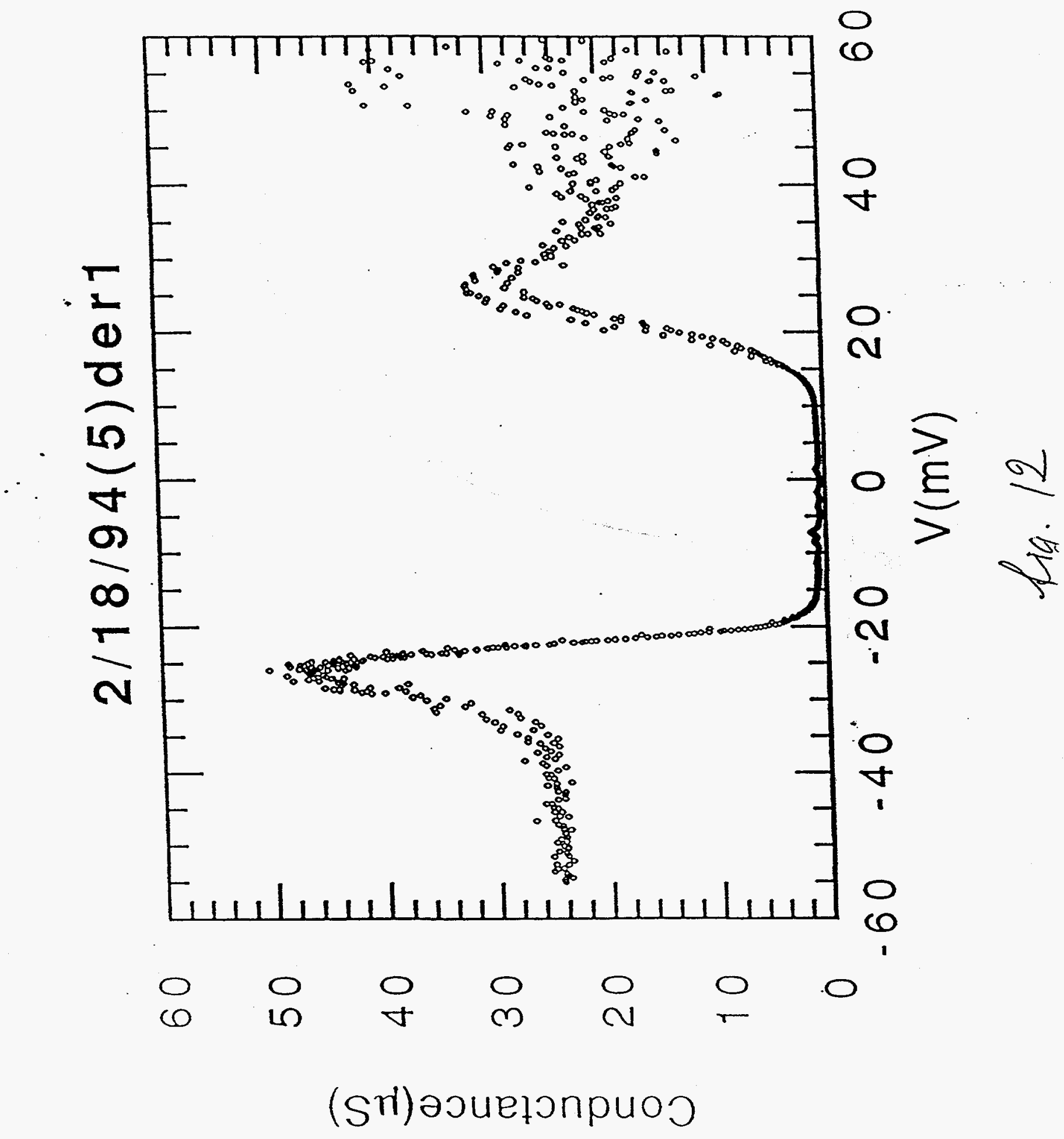

